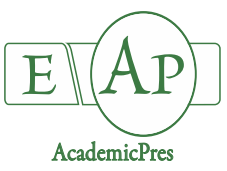

Tassoula L et al. (2021)

Notulae Botanicae Horti Agrobotanici Cluj-Napoca

Volume 49, Issue 2, Article number 12283

DOI: $10.15835 /$ nbha 49212283

Research Article

\title{
Water use efficiency, growth and anatomic-physiological parameters of Mediterranean xerophytes as affected by substrate and irrigation on a green roof
}

\author{
Lamprini TASSOULA ${ }^{1}$, Maria PAPAFOTIOU ${ }^{1 *}$, \\ Georgios LIAKOPOULOS², George KARGAS ${ }^{3}$ \\ ${ }^{1}$ Agricultural University of Athens, School of Plant Sciences, Department of Crop Science, Laboratory of Floriculture and Landscape
Architecture, Iera Odos 75, 11855 Athens, Greece; lamprinitasoula@gmail.com; mpapaf@aua.gr (*corresponding author)
${ }^{2}$ Agricultural University of Athens, School of Plant Sciences, Department of Crop Science, Laboratory of Plant Physiology and
Morphology, Iera Odos 75, 11855 Athens, Greece; gliak@aua.gr
${ }^{3}$ Agricultural University of Athens, School of Environment and Agricultural Engineering, Department of Natural Resources
Development and Agricultural Engineering, Laboratory of Agricultural Hydraulics, Iera Odos 75, 11855 Athens, Greece;
kargas@aua.gr
}

Abstract

The water use efficiency, growth and anatomic-physiological parameters of Mediterranean medicinal xerophytes on a green roof was investigated, under the effect of water stress and different types of substrate. Rooted cuttings of Convolvulus cneorum, Origanum dictamnus, Sideritis athoa, Atriplex halimus and Lomelosia cretica were planted on a roof in Athens, Greece. Two substrate types, $10 \mathrm{~cm}$ deep, were used, i.e. grape marc compost: perlite: soil: pumice $(3: 3: 2: 2, \mathrm{v} / \mathrm{v})$ and a lighter one, i.e. grape marc compost: perlite: pumice $(3: 3: 4, \mathrm{v} / \mathrm{v})$. Two irrigation frequencies were applied during the dry period (summer), normal and sparse, when substrate moisture was $17-20 \%$ and 5-11\%, respectively. Water use efficiency was increased by sparse irrigation in $A$. halimus and L. cretica and by soil substrate in $C$. cneorum. Sparse irrigation reduced dry weight in $O$. dictamnus, $S$. athoa and L. cretica and in $A$. halimus in combination with soilless substrate. It increased leaf thickness in all species and reduced $\mathrm{Ch}_{\circ \lambda}$ in all species, other than $S$. athoa, while in C. cneorum, $O$. dictamnus and L. cretica this was evident only in the presence of soil in the substrate. In summer, one $\mathrm{d}$ before irrigation, stomatal resistance $\left(\mathrm{R}_{\text {leaf }}\right)$ was increased and maximum quantum yield of PSII ( $\left.\Phi_{\text {PSIIo }}\right)$ decreased indicating water limitation, while both parameters were restored to normal levels one $\mathrm{d}$ after irrigation indicating that both can serve as sensitive indicators of the onset of water stress. $\Phi_{\text {PSIIo }}$ values, never fell to critical levels, excluding irreversible damage to the photosynthetic apparatus. Therefore, all five plant species are recommended for use on extensive green roofs in semi-arid areas.

Keywords: Atriplex halimus; Convolvulus cneorum; Lomelosia cretica; medicinal plants; Origanum dictamnus; Sideritis athoa

Received: 18 Feb 2021. Received in revised form: 24 Mar 2021. Accepted: 12 Apr 2021. Published online: 29 Apr 2021.

From Volume 49, Issue 1, 2021, Notulae Botanicae Horti Agrobotanici Cluj-Napoca journal uses article numbers in place of the traditional method of continuous pagination through the volume. The journal will continue to appear quarterly, as before, with four annual numbers. 


\section{Introduction}

The indisputable fact of global warming causing constant climate change (Nila et al., 2019; IPCC, 2014; 2007) leads to vegetation changes (Sangüesa-Barreda et al., 2019) and predicts that the next 30 - 90 years, there will be severe and extensive droughts due to water scarcity and increased evaporation (Dai, 2013). Hence, environmental strategies, such as "green constructions" and "green networks", will play a dynamic role in the urban environment, living standards and public health (Cameron et al., 2012; Santamouris, 2014; Kondo et al., 2018; Abass et al., 2020; Feitosa and Wilkinson, 2020; Cristiano et al., 2021). In recent decades, green roofs are the favorite scientific subject of many researchers as a climate adaptation strategy (Andric et al., 2020) and as a dynamic and ecological approach for degraded urban areas (Dunnett and Kingsbury, 2008; Papafotiou et al., 2013; Vijayaraghavan, 2016; Moore et al., 2017; Silva et al., 2018), promoting human, social and environmental sustainability.

Urban culture in private or in public green roofs, is a rational and feasible solution for economical growth, so their techniques and construction materials should be improved and given to the general public (Whittinghill and Rowe, 2012). The design of the green roofs should be based on qualitative and quantitative criteria and should be modified according to the needs of the citizens (Langemeyer et al., 2020). Experiments on the production of herbs and vegetables in extensive green roofs, especially in shallow substrates, have given positive results, either as a complementary source of food production (Walters and Stoelzle Midden, 2018) or helping to manage rainwater in quantity or quality (Whittinghill et al., 2013; 2015; Liu et al., 2021).

In experiments on Mediterranean plant species, a wide range of water use efficiency (WUE) values was observed, which may be due to, water management, plant species, stage of development, as well as environmental factors. Therefore, the study and the improvement of WUE in the Mediterranean should be explored in more detail, considering the lack of water resources and the growth of human population (Katerji et al., 2008). WUE could be maintained at high levels by choosing the right plant species and with the application of the right irrigation methods (Hatfield and Dold, 2019). Deficit irrigation achieves increased WUE without causing serious reduction in yield for various crops (Geerts and Raes, 2009) and microirrigation plays an important role in this (Fan et al., 2018). After all, in green roofs where production is not the first goal of using a plant species, the application of deficit irrigation is even more encouraging.

The substrate materials determine the weight of the green roof construction, which is an important issue in the treatment of old Mediterranean buildings. Due to the fact that soil is heavy for use, researchers are experimenting on creating light substrates (Beattie and Berghage, 2004) often without soil involvement with positive results (Eksi and Rowe, 2019). The participation of organic matter in the substrate helps to retain moisture and provides nutrients to plants. Therefore, the use of compost in green roofs is investigated repeatedly and, in many ways, (Reis et al., 2001; Santos et al., 2008; Tassoula et al., 2015; Papafotiou et al., 2016; Jusselme et al., 2019; Xue and Farrell, 2020). It has recently been shown that the participation of a compost in combination with earthworms apart from promoting plant growth it induced an increase of nectar volume in flowers and in sugars concentration that resulted in greater approach of various and abundant pollinators (Jusselme et al., 2019).

In green roofs, at the eastern Mediterranean that is characterized by intense sunlight and several periods of drought, xerophytes show successful establishment and enhanced growth and development (Papafotiou et al., 2012; 2013; Tassoula et al., 2015; Martinetti et al., 2018). Their acclimation to water stress involves important structures such as pubescence (Karabourniotis et al., 2020a) and calcium oxalate crystals or amorphous calcium carbonate cystoliths (Karabourniotis et al., 2020b). Native plants maintain and increase biodiversity, including pollinators and habitats (Lundholm and Peck, 2008; Cook-Patton and Bauerle, 2012; Benvenuti, 2014), while protecting local character, giving the image of a natural landscape and improving the psychology of employees in their workplace (Loder, 2014). Mediterranean flora includes a very large number of aromatic and medicinal plant species, 500-600 in Greece. Although the climate is ideal for growing many of the above plants, their cultivation is limited and is mainly of local interest. In addition, aromatic and medicinal 
plants have low water, nutrient and maintenance requirements and high resistance to the polluted urban environment while having high aesthetic value and they attract insects, especially pollinators for their rich pollen.

Convolvulus cneorum L. is used to produce alkaloids in medicine (Palazón et al., 2006) and has been shown to be very effective in curbing particulate air pollution (Shackleton et al., 2012). Origanum dictamnus L. known from ancient times, was used for diseases of the stomach and in general the digestive system, spleen, for rheumatism, arthritis and finally for uterine diseases and dystocia (Liolios, 2009). Sideritis athoa L. is a powerful antioxidant (Gonzalez-Burgos et al., 2011) and acts against viruses, yeasts and bacteria through its essential oils, mainly apipene and carvacrol (Aligiannis et al., 2001; Fokialakis et al., 2007). Atriplex halimus L. is used to treat heart disease, diabetes and rheumatism (Said et al., 2002, 2008). Finally, Lomelosia cretica(L.) Greuter and Burdet contains vitamin K1, C, lutein, tocopherol etc. that improve human health and contribute to its recovery (Vardavas, 2006) and fatty acids and sterol, which give it antibacterial properties (Perdetzoglou et al., 1996).

In the present work research was undertaken on the combined effect of substrate type and irrigation frequency on water use efficiency, growth and anatomical and physiological parameters of the five Mediterranean medicinal xerophytes mentioned above, in an extensive type green roof in Athens, Greece, aiming to a sustainable green roof system under harsh environmental conditions.

\section{Materials and Methods}

\section{Experimental setup}

The experiments were performed on a second floor, fully exposed flat roof at the Agricultural University of Athens (37 59' N, 2342' E) from July 152011 to October 152013 (Convolvulus cneorum, Origanum dictamnus. and Sideritis athoa) and from February 152013 to September 152014 (Atriplex halimus and Lomelosia cretica). For the first three species, $C$. cneorum, $O$. dictamnus. and $S$. athoa, at the site of the experiments a green roof infrastructure was installed, which included substrate moisture retention and protection of the insulation mat (TSM32; Zinco, Egreen, Athens, Greece) and a drainage element (FD25; Zinco, Egreen), as described by Tassoula et al. (2015). The roof was divided with planks to square modules of $50 \mathrm{~cm}$, and in each module the drainage layer was covered by a filter sheet of non-woven geotextile (SF; Zinco, Egreen). On top of it was placed the plant growing substrate. Two rooted cuttings of either $C$. cneorum, or $O$. dictamnus, or $S$. athoa, were planted diagonally in each experimental module. The rooted cuttings of $A$. halimus and $L$. cretica were planted in plastic containers, $60 \mathrm{~cm}$ long and $40 \mathrm{~cm}$ wide, with the same as above green roof infrastructure fitted. Two types of substrate mix of $10 \mathrm{~cm}$ depth were used; one including soil and a soilless, that were made of grape marc compost: perlite: soil: pumice (3:3:2:2, $\mathrm{v} / \mathrm{v})$ and grape marc compost: perlite: pumice $(3: 3: 4, \mathrm{v} / \mathrm{v})$, respectively.

\section{Irrigation}

The irrigation schedule was applied each dry period from the beginning of May to end of September, by automatic drip irrigation on the surface of the growth substrate, before sunrise, by two drippers placed at equal distances from the center of the container and the two plants (dripper supply $4 \mathrm{~L} \mathrm{~h}^{-1}$ ). Each irrigation event lasted $60 \mathrm{~min}$ to allow water to run off the container. The first week after planting, irrigation was applied every $2 \mathrm{~d}$ to those that were planted in summer 2011 and every $4 \mathrm{~d}$ to those that were planted in winter 2013, in order for the plants to overcome transplant stress. On July 22, 2011 (C. cneorum, O. dictamnus and S. athoa) and on May 15, 2013 (A. halimus and L. cretica), plants were irrigated and then were exposed to a preliminary drought experiment for determining the number of $d$ that the plants could withstand between two irrigation events. The daily measurements of the substrate water content $(\% \mathrm{v} / \mathrm{v})$ recorded as described by Tassoula et al. (2015) and the wilting symptoms of each species determined the sparse irrigation frequency. Two irrigation 
frequencies were applied during the dry period (May-September), normal and sparse, when substrate moisture was $17-20 \%$ and $5-11 \%$, respectively. Substrate water content tests were carried out regularly until the end of September of each year when irrigation stopped. Overall, four treatments were applied (two substrate types combined with two irrigation frequencies). Four modules (containers) per treatment were used for $C$. cneorum, $O$. dictamnus and $S$. athoa and six for A. halimus and L. cretica, with two plants planted in each module. Based on the above, in the experiments of $C$. cneorum, $O$. dictamnus and $S$. athoa, during the dry period of 2011 and 2012, irrigation was applied either every $5 \mathrm{~d}$ (normal) or every $7 \mathrm{~d}$ (sparse), while during the dry period of 2013 normal irrigation was determined at $4 \mathrm{~d}$ and sparse at 6 , as the plants were larger and had higher water needs. In the experiment of $L$. cretica irrigation during the dry period was applied either every $3 \mathrm{~d}$ (normal) or every $5 \mathrm{~d}$ (sparse) in 2013 and 2014 with the exception of August 2014 where normal irrigation was determined at $2 \mathrm{~d}$ and sparse at $4 \mathrm{~d}$. In the experiment of $A$. halimus during the dry season 2013, normal irrigation was applied every $5 \mathrm{~d}$ and every $9 \mathrm{~d}$ the sparse, while during the dry season 2014, normal irrigation was applied every 4 and every $7 \mathrm{~d}$, respectively.

\section{Meteorological data}

The meteorological data (average monthly air temperature, total monthly rainfall, and average relative humidity for the water stress periods of the experiment, Figure 1) collected from a meteorological cage located near the experimental site, were provided by the Laboratory of General and Agricultural Meteorology of the Agricultural University of Athens.

\section{Chemical and physical properties of the substrates}

The physicochemical properties of the substrates and their elements, as well as the moisture retention curves of the substrates have been described by Tassoula et al. (2015). Some further information on $\mathrm{pH}$ and Electrical Conductivity (EC) at the begging and the end of the experimental period is provided in Figure 2. The EC was measured in 1:5 (EC $\left(\mathrm{EC}_{1: 5}\right)$ substrate over water ratio where $50 \mathrm{ml}$ of substrate and $250 \mathrm{~mL}$ of distilled water were used. The suspension is shaken by hand, 4 times, every $0.5 \mathrm{~h}$ for $30 \mathrm{~s}$. After, the extract was obtained, and the EC was determined.

\section{Growth evaluation}

The dry weight of the aboveground part of the plants was measured after oven-drying at $70{ }^{\circ} \mathrm{C}$ for $8 \mathrm{~d}$. The increase in plant diameter was calculated by subtracting from the final plant diameter the initial. As plant diameter the average of the biggest diameter and its perpendicular was used.

\section{Water use efficiency evaluation}

WUE was calculated from the quotient of the dry weight of the aboveground part of each plant to its total water consumption (supplied by irrigation) during the experiment (Table 3). Total water consumption of each plant was calculated from the amount of water added in each irrigation event, multiplied by the number of irrigations events during the experiment.

\section{Total chlorophyll concentration (Chl tot $)$ evaluation}

Concentration of chlorophylls was measured photometrically. Optical density of $80 \%$ acetone extracts of leaf disks was measured at 663, 647 and $720 \mathrm{~nm}$ in a UV 160A dual beam spectrophotometer (Shimadzu Co., Tokyo, Japan) according to Lichtenthaler (1987).

\section{Determination of leaf thickness}

The thickness of the leaf was measured under an optical microscope (Zeiss Axiolab, Carl Zeiss, Jena, Germany), from cross sections of fresh leaves taken from the middle of the leaf lamina, with a razor blade. Five leaves per plant, fully exposed to the sun, were selected from four plants per treatment. 
Leaf stomatal resistance $\left(R_{\text {leaf }}\right)$ evaluation

During the periods of water stress application (summer periods) and once during the winter, $\mathrm{R}_{\text {leaf }}$ was recorded with an AP4 Porometer (Delta-T devices) on two fully developed young leaves of each plant from $11.00-13.00_{\mathrm{HR}}$, as defined by the daily fluctuation of $\mathrm{R}_{\text {leaf. }}$

Maximum quantum yield of PSII photochemistry $\left(\Phi_{\text {PSIIo }}\right)$ evaluation

$\Phi_{\text {PSIIo }}$ was measured in all plants during the periods of water stress application and each February, the d before and the $\mathrm{d}$ after the irrigation events, with a Photosynthesis Yield Analyzer (MINI-PAM, Portable Fluorometer, Walz, Effeltrich, Germany). Eight to twelve measurements per treatment were taken as described by Tassoula et al. (2015).

\section{Observation / illustration of leaf blade structure}

In mid-July, leaves were collected from all plant species, fully developed, of the same orientation and exposure to the sun and from all operations. In the Electron Microscopy Laboratory, the selected sections were solidified with a suitable cooling gel (Jung, Tissue freezing medium, Leica, Germany) in special metal samplers and placed in the refrigeration microtome (Kryotom, Leica CM1850, Germany), at a temperature of $-20^{\circ} \mathrm{C}$. Sections were observed in the optical microscope (Olympus BX 40 Olympus, Japan) through the CellA program (Soft Imagine Systems, Olympus cell family, Japan), while photographing was taken with the Olympus DP71 digital camera, with a resolution of $12.5 \mathrm{Mp}$ in the visible spectrum.

\section{Statistical analysis}

Five multifactorial experiments, one for each plant species, with two factors each were conducted. The two factors were: substrate type (soil and soilless), irrigation frequency (normal and sparse, when substrate moisture was $17-20 \%$ and $5-11 \%$, respectively). Therefore, four treatments were applied ( 2 substrates $\mathrm{x} 2$ irrigations). The containers were arranged following the completely randomized design. The significance of the results was tested by two-way analysis of variance (ANOVA) ( $F$ test, discrete variables followed the normal distribution). The treatment means were compared using Fisher's Least Significant Difference (LSD) or Student's $t$ at $P \leq 0.05$. JMP version 11 statistical software (SAS Institute Inc., Cary, NC) was used.

\section{Results}

\section{Meteorological data}

The meteorological data for the periods May to end of September that plants were exposed to deficient irrigation treatment are shown in Figure 1. During this period there were very few incidents of rain, especially in July and August, and they did not affect irrigation treatments.

\section{Chemical properties of substrates}

The $\mathrm{EC}_{1: 5}$ of the substrates, was initially elevated due to the presence of compost, but over time, a large decrease was observed (Figure 2). 


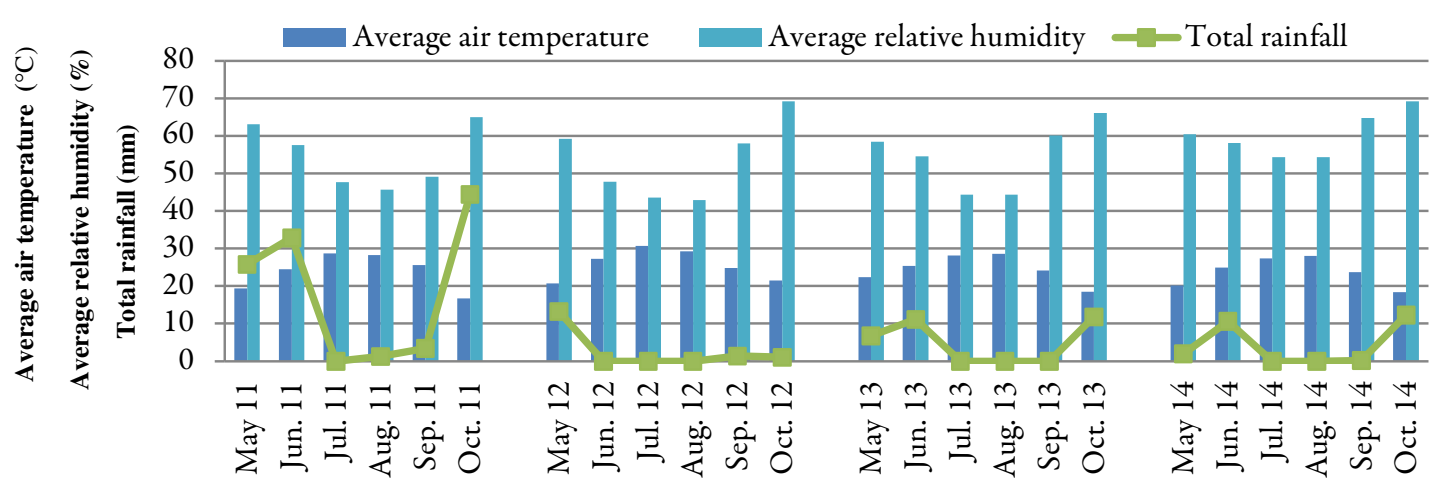

Date (month)

Figure 1. The average monthly air temperature, total monthly rainfall, and average relative humidity for the water stress periods of the experiment (May - Oct. 2011 - 2014)
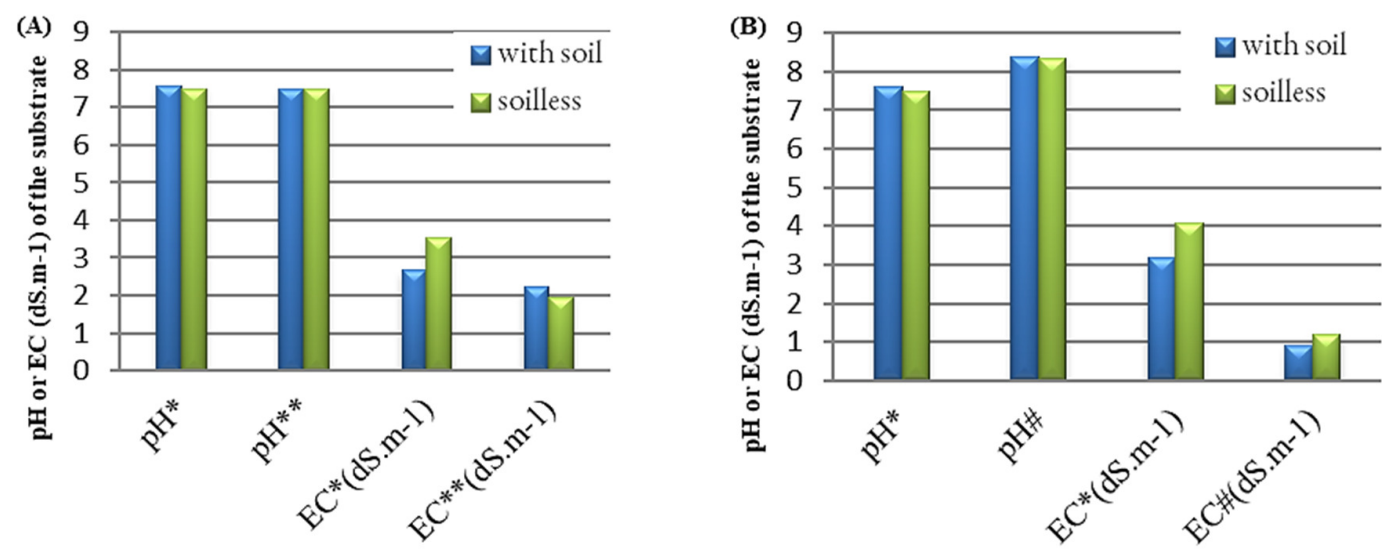

Figure 2. $\mathrm{Ph}$ and $\mathrm{EC}_{1: 5}$ values of the substrates, in experiments with $C$. cneorum, $O$. dictamnus and $S$. athoa (A) and A. halimus and L. cretica (B), at planting $\left(^{*}\right)$ and after $\left(^{* *}\right) 27$ or $10(\#)$ months of culture in (A) or (B) respectively

\section{Plant growth}

Sparse irrigation adversely affected dry weight in O. dictamnus, S. athoa and L. cretica and in A. halimus in combination with soilless substrate, while in $C$. cneorum the soilless substrate had a negative effect (Table 1). The same happened with the increase of the diameter, except for the $S$. athoa that there was no effect of the main factors (Table 2). However, $S$. athoa's plants grown in the soil substrate presented a greater increase in diameter when irrigated normally (Table 2).

\section{Water use efficiency}

Water use efficiency was increased by sparse irrigation in $A$. halimus and L. cretica and by soil substrate in $C$. cneorum, while it was not affected by the main factors in $O$. dictamnus and $S$. athoa (Table 3). 
Table 1. The effect of the main experimental factors, i.e., irrigation frequency, normal (n) or sparse (s) and substrate type (with soil: $3 \mathrm{GC}: 3 \mathrm{P}: 2 \mathrm{~S}: 2 \mathrm{Pu}$ or soilless: $3 \mathrm{GC}: 3 \mathrm{P}: 4 \mathrm{Pu}$ ), and the effect of the experimental treatments, on above ground dry weight $(\mathrm{g})$ of each plant species at the end of the experimental period (Jul. 2011- Oct. 2013 for C. cneorum, O. dictamnus and S. athoa; Feb. 2013 - Sept. 2014 for A. halimus and L. cretica)

\begin{tabular}{|c|c|c|c|c|c|}
\hline Main factor ${ }^{2}$ & $\begin{array}{l}\text { C. cneorum } \\
\text { Oct. } 2013\end{array}$ & $\begin{array}{c}\text { O. dictamnus } \\
\text { Oct. } 2013\end{array}$ & $\begin{array}{c}\text { S. athoa } \\
\text { Oct. } 2013\end{array}$ & $\begin{array}{l}\text { A. halimus } \\
\text { Sept. } 2014\end{array}$ & $\begin{array}{c}\text { L. cretica } \\
\text { Sept. } 2014\end{array}$ \\
\hline Normal & $208.8 \pm 17,5 \mathrm{a}$ & $112.0 \pm 10.1 \mathrm{a}$ & $257.5 \pm 28.9 \mathrm{a}$ & $186.2 \pm 5.8 \mathrm{a}$ & $127.2 \pm 6.0 \mathrm{a}$ \\
\hline Sparse & $193.6 \pm 21.4 \mathrm{a}$ & $62.2 \pm 8.5 b$ & $171.2 \pm 28.9 b$ & $136.0 \pm 6.7 \mathrm{~b}$ & $96.6 \pm 5.2 \mathrm{~b}$ \\
\hline Soil & $259.1 \pm 17.5 \mathrm{a}$ & $95.6 \pm 10.3 \mathrm{a}$ & $207.6 \pm 26.8 \mathrm{a}$ & $172.4 \pm 6.3 \mathrm{a}$ & $112.9 \pm 5.6 \mathrm{a}$ \\
\hline Soilless & $143.3 \pm 21.4 b$ & $78.5 \pm 8.5 \mathrm{a}$ & $221.2 \pm 30.9 \mathrm{a}$ & $149.8 \pm 6.1 b$ & $110.9 \pm 5.6 \mathrm{a}$ \\
\hline Firrig & $n s$ & $*$ & * & * & $*$ \\
\hline Fsubst & $*$ & $n s$ & $n s$ & $*$ & $n s$ \\
\hline Fir xs & $n s$ & $n s$ & $n s$ & $n s$ & $n s$ \\
\hline \multicolumn{6}{|l|}{ Treatment $^{\mathrm{y}}$} \\
\hline Soil/n & $275.9 \pm 28,4 \mathrm{a}$ & $139.7 \pm 17.2 \mathrm{a}$ & $282.2 \pm \mathrm{a}$ & $204.3 \pm 7.8 \mathrm{a}$ & $134.2 \pm 8.5 \mathrm{a}$ \\
\hline Soil/s & $242.4 \pm 31,9 \mathrm{a}$ & $51.60 \pm 11.3 \mathrm{c}$ & $133.0 \pm b$ & $140.4 \pm 10.1 \mathrm{c}$ & $91.6 \pm 7.4 \mathrm{c}$ \\
\hline Soilless/n & $141.7 \pm 15,5 b$ & $84.10 \pm 10.6 b$ & $232.8 \pm \mathrm{ab}$ & $168.1 \pm 8.7 b$ & $120.2 \pm 8.5 \mathrm{ab}$ \\
\hline Soilless/s & $144.9 \pm 12,0 \mathrm{~b}$ & $72.80 \pm 13.4 \mathrm{bc}$ & $209.5 \pm \mathrm{ab}$ & $131.6 \pm 8.7 \mathrm{c}$ & $101.6 \pm 7.4 \mathrm{bc}$ \\
\hline
\end{tabular}

${ }^{z}$ Mean $\left( \pm\right.$ SE) comparison in columns within each main factor with Student's $t$ at $P \leq 0.05$; ${ }^{\mathrm{P}}$ Mean $( \pm \mathrm{SE})$ comparison in columns within treatments with Student's $t$ at $P \leq 0.05$; Means followed by the same letter are not significantly different at $P \leq 0.05 ;^{*}=$ significant at $P \leq 0.05 ;$ ns $=$ not significant

Table 2. The effect of the main experimental factors, i.e., irrigation frequency, normal (n) or sparse (s) and substrate type (with soil: $3 \mathrm{GC}: 3 \mathrm{P}: 2 \mathrm{~S}: 2 \mathrm{Pu}$ or soilless: $3 \mathrm{GC}: 3 \mathrm{P}: 4 \mathrm{Pu}$ ), and the effect of the experimental treatments on diameter increase $(\mathrm{cm})$ of each plant species during the experimental period (Jul. 2011- Oct. 2013 for C. cneorum, O. dictamnus and S. athoa; Feb. 2013 - Sept. 2014 for A. halimus and L. cretica)

\begin{tabular}{|l|c|c|c|c|c|}
\hline \multicolumn{1}{|c|}{ Main factor ${ }^{2}$} & C. cneorum & O. dictamnus & S. athoa & A. halimus & L. cretica \\
\hline Normal & $91.2 \pm 4.9 \mathrm{a}$ & $45.1 \pm 1.3 \mathrm{a}$ & $49.3 \pm 4.4 \mathrm{a}$ & $102.9 \pm 5.1 \mathrm{a}$ & $28.5 \pm 0.9 \mathrm{a}$ \\
\hline Sparse & $86.2 \pm 5.3 \mathrm{a}$ & $34.6 \pm 1.3 \mathrm{~b}$ & $44.7 \pm 5.3 \mathrm{a}$ & $86.3 \pm 5.9 \mathrm{~b}$ & $25.6 \pm 0.9 \mathrm{~b}$ \\
\hline Soil & $97.6 \pm 4.9 \mathrm{a}$ & $41.7 \pm 1.3 \mathrm{a}$ & $45.2 \pm 4.8 \mathrm{a}$ & $103.0 \pm 5.6 \mathrm{a}$ & $26.6 \pm 0.9 \mathrm{a}$ \\
\hline Soilless & $79.9 \pm 5.3 \mathrm{~b}$ & $38.0 \pm 1.3 \mathrm{a}$ & $48.7 \pm 5.0 \mathrm{a}$ & $86.2 \pm 5.3 \mathrm{~b}$ & $27.5 \pm 0.9 \mathrm{a}$ \\
\hline Firrig & $n s$ & ${ }^{*}$ & $n s$ & ${ }^{*}$ & $n{ }^{*}$ \\
\hline Fsubst & $*$ & $n s$ & $n s$ & ${ }^{*}$ & $n s$ \\
\hline Fir x s & $n s$ & $n s$ & $n s$ & $n s$ & $n s$ \\
\hline Treatment & & & & & $28.5 \pm 1.3 \mathrm{a}$ \\
\hline Soil/n & $102.7 \pm 8.4 \mathrm{a}$ & $49.6 \pm 1.2 \mathrm{a}$ & $55.8 \pm 5.9 \mathrm{a}$ & $126.6 \pm 7.1 \mathrm{a}$ & $28.7 \pm 1.3 \mathrm{~b}$ \\
\hline Soil/s & $92.4 \pm 8.3 \mathrm{ab}$ & $33.9 \pm 1.6 \mathrm{c}$ & $34.7 \pm 7.6 \mathrm{~b}$ & $79.4 \pm 8.7 \mathrm{~b}$ & $24.7 \pm 1.3 \mathrm{a}$ \\
\hline Soilless/n & $79.6 \pm 5.9 \mathrm{~b}$ & $40.7 \pm 1.8 \mathrm{~b}$ & $42.7 \pm 6.5 \mathrm{ab}$ & $93.2 \pm 7.1 \mathrm{~b}$ & $28.5 \pm 1.3 \mathrm{ab}$ \\
\hline Soilless/s & $80.0 \pm 3.9 \mathrm{~b}$ & $35.4 \pm 2.7 \mathrm{bc}$ & $54.7 \pm 7.6 \mathrm{ab}$ & $79.2 \pm 7.8 \mathrm{~b}$ & $26.5 \pm 1.3 \mathrm{ab}$ \\
\hline
\end{tabular}

${ }^{7}$ Mean $\left( \pm\right.$ SE) comparison in columns within each main factor with Student's $t$ at $P \leq 0.05$; ${ }^{\mathrm{y}}$ Mean $( \pm \mathrm{SE})$ comparison in columns within treatments with Student's $t$ at $P \leq 0.05$; Means followed by the same letter are not significantly different at $P \leq 0.05 ;{ }^{*}=$ significant at $P \leq 0.05 ; n s=$ not significant 
Table 3. The effect of the main experimental factors, i.e., irrigation frequency, normal (n) or sparse (s) and substrate type (with soil: $3 \mathrm{GC}: 3 \mathrm{P}: 2 \mathrm{~S}: 2 \mathrm{Pu}$ or soilless: $3 \mathrm{GC}: 3 \mathrm{P}: 4 \mathrm{Pu}$ ), and the effect of the experimental treatments, on water use efficiency (WUE) of each plant species at the end of the experimental period (Jul. 201 - Oct. 2013 for C. cneorum, O. dictamnus and S. athoa; Feb. 2013 - Sept. 2014 for A. halimus and $L$. cretica)

\begin{tabular}{|c|c|c|c|c|c|}
\hline Main factor ${ }^{2}$ & $\begin{array}{l}\text { C. cneorum } \\
\text { Oct. } 2013\end{array}$ & $\begin{array}{c}\text { O. dictamnus } \\
\text { Oct. } 2013\end{array}$ & $\begin{array}{c}\text { S. athoa } \\
\text { Oct. } 2013\end{array}$ & $\begin{array}{l}\text { A. halimus } \\
\text { Sept. } 2014\end{array}$ & $\begin{array}{c}\text { L. cretica } \\
\text { Sept. } 2014\end{array}$ \\
\hline Normal & $0.61 \pm 0.06 \mathrm{a}$ & $0.33 \pm 0.03 \mathrm{a}$ & $0.76 \pm 0.16 \mathrm{a}$ & $0.9 \pm 0.04 \mathrm{~b}$ & $0.29 \pm 0.02 b$ \\
\hline Sparse & $0.79 \pm 0.06 \mathrm{a}$ & $0.26 \pm 0.03 \mathrm{a}$ & $0.71 \pm 0.14 \mathrm{a}$ & $1.30 \pm 0.05 \mathrm{a}$ & $0.38 \pm 0.02 \mathrm{a}$ \\
\hline Soil & $0.91 \pm 0.06 \mathrm{a}$ & $0.31 \pm 0.03 \mathrm{a}$ & $0.69 \pm 0.13 \mathrm{a}$ & $1.15 \pm 0.05 \mathrm{a}$ & $0.34 \pm 0.02 \mathrm{a}$ \\
\hline Soilless & $0.50 \pm 0.06 b$ & $0.27 \pm 0.03 \mathrm{a}$ & $0.77 \pm 0.17 \mathrm{a}$ & $1.08 \pm 0.05 \mathrm{a}$ & $0.34 \pm 0.02 \mathrm{a}$ \\
\hline Firrig & ns & ns & $n s$ & ${ }^{*}$ & ${ }^{*}$ \\
\hline Fsubst & $*$ & $n s$ & $n s$ & $n s$ & $n s$ \\
\hline Fir xs & $n s$ & $n s$ & $n s$ & $n s$ & $n s$ \\
\hline \multicolumn{6}{|l|}{ Treatment $^{y}$} \\
\hline Soil/n & $0.81 \pm 0.09 \mathrm{ab}$ & $0.41 \pm 0.06 \mathrm{a}$ & $0.83 \pm 0.18 \mathrm{a}$ & $0.97 \pm 0.06 b$ & $0.31 \pm 0.03 \mathrm{bc}$ \\
\hline Soil/s & $1.01 \pm 0.09 \mathrm{a}$ & $0.21 \pm 0.04 b$ & $0.55 \pm 0.18 \mathrm{a}$ & $1.33 \pm 0.07 \mathrm{a}$ & $0.36 \pm 0.02 \mathrm{ab}$ \\
\hline Soilless/n & $0.42 \pm 0.09 \mathrm{c}$ & $0.25 \pm 0.04 \mathrm{~b}$ & $0.68 \pm 0.26 a$ & $0.89 \pm 0.06 b$ & $0.28 \pm 0.03 c$ \\
\hline Soilless/s & $0.57 \pm 0.09 \mathrm{bc}$ & $0.30 \pm 0.04 \mathrm{ab}$ & $0.87 \pm 0.21 \mathrm{a}$ & $1.26 \pm 0.06 \mathrm{a}$ & $0.40 \pm 0.02 \mathrm{a}$ \\
\hline
\end{tabular}

${ }^{z}$ Mean $( \pm S E)$ comparison in columns within each main factor with Student's $t$ at $P \leq 0.05$; ${ }^{\mathrm{y}} \mathrm{Mean}( \pm \mathrm{SE})$ comparison in columns within treatments with Student's $t$ at $P \leq 0.05$; Means followed by the same letter are not significantly different at $P \leq 0.05 ;{ }^{*}=$ significant at $P \leq 0.05 ;$ ns $=$ not significant



Sparse irrigation also reduced $\mathrm{Chl}_{\diamond \lambda}$ of all plant species, other than $S$. athoa, while in $C$. cneorum, $O$. dictamnus and L. cretica this effect was evident only in the presence of soil in the substrate. In C. cneorum, O. dictamnus and $S$. athoa the combination of soilless substrate and sparse irrigation led to lower total chlorophyll concentrations (Table 4).

Table 4. The effect of the main experimental factors, i.e., irrigation frequency, normal (n) or sparse ( $s$ ) and substrate type (with soil: $3 \mathrm{GC}: 3 \mathrm{P}: 2 \mathrm{~S}: 2 \mathrm{Pu}$ or soilless: $3 \mathrm{GC}: 3 \mathrm{P}: 4 \mathrm{Pu}$ ), and the effect of the experimental treatments, on total chlorophyll concentration $\left(\mathrm{Chl}_{\text {tot }}\right)$ in the middle of the dry season (July 2013 or 2014)

\begin{tabular}{|c|c|c|c|c|c|}
\hline Main factor ${ }^{2}$ & $\begin{array}{c}\text { C. cneorum } \\
\text { July } 2013\end{array}$ & $\begin{array}{l}\text { O. dictamnus } \\
\text { July } 2013\end{array}$ & $\begin{array}{c}\text { S. athoa } \\
\text { July } 2013\end{array}$ & $\begin{array}{l}\text { A. halimus } \\
\text { July } 2014\end{array}$ & $\begin{array}{l}\text { L. cretica } \\
\text { July } 2014\end{array}$ \\
\hline Normal & $30.2 \pm 2.3 \mathrm{a}$ & $24.9 \pm 2.08 \mathrm{a}$ & - & $38.0 \pm 1.7 \mathrm{a}$ & $12.4 \pm 6.1 \mathrm{a}$ \\
\hline Sparse & $16.1 \pm 2.3 b$ & $13.0 \pm 2.08 \mathrm{~b}$ & - & $17.7 \pm 1.7 b$ & $3.4 \pm 6.1 \mathrm{~b}$ \\
\hline Soil & $26.6 \pm 2.3 \mathrm{a}$ & $23.5 \pm 2.08 \mathrm{a}$ & - & $28.4 \pm 1.7 \mathrm{a}$ & $12.4 \pm 6.1 \mathrm{a}$ \\
\hline Soilless & $19.7 \pm 2.3 b$ & $14.5 \pm 2.08 \mathrm{~b}$ & - & $27.2 \pm 1.7 \mathrm{a}$ & $3.4 \pm 6.1 \mathrm{~b}$ \\
\hline Firrig & $*$ & $*$ & - & $*$ & $*$ \\
\hline Fsubst & $*$ & * & - & $n s$ & $*$ \\
\hline Fir x s & $n s$ & $n s$ & $*$ & ns & $n s$ \\
\hline \multicolumn{6}{|l|}{ Treatment $^{y}$} \\
\hline Soil/n & $29.5 \pm 3.2 \mathrm{a}$ & $27.4 \pm 2.9 \mathrm{a}$ & $23.7 \pm 3.1 \mathrm{a}$ & $38.7 \pm 2.4 \mathrm{a}$ & $20.8 \pm 8.6 \mathrm{a}$ \\
\hline Soil/s & $23.7 \pm 3.2 \mathrm{a}$ & $19.6 \pm 2.9 \mathrm{a}$ & $26.3 \pm 3.1 \mathrm{a}$ & $18.0 \pm 2.4 \mathrm{~b}$ & $3.9 \pm 8.6 \mathrm{~b}$ \\
\hline Soilless/n & $30.0 \pm 3.2 \mathrm{a}$ & $22.4 \pm 2.9 \mathrm{a}$ & $27.8 \pm 3.1 \mathrm{a}$ & $37.0 \pm 2.4 \mathrm{a}$ & $4.0 \pm 8.6 \mathrm{~b}$ \\
\hline Soilless/s & $8.4 \pm 3.2 \mathrm{~b}$ & $6.5 \pm 2.9 \mathrm{~b}$ & $4.9 \pm 3.1 \mathrm{~b}$ & $17.3 \pm 2.4 \mathrm{~b}$ & $2.8 \pm 8.6 \mathrm{~b}$ \\
\hline
\end{tabular}

${ }^{z}$ Mean $( \pm \mathrm{SE})$ comparison in columns within each main factor with Student's $t$ at $P \leq 0.05$; ${ }^{\mathrm{y}}$ Mean $( \pm \mathrm{SE})$ comparison in columns within treatments with Student's $t$ at $P \leq 0.05$; Means followed by the same letter are not significantly different at $P \leq 0.05 ;^{*}=$ significant at $P \leq 0.05 ; n s=$ not significant 


\section{Leaf thickness}

The thickness of the leaf was increased by sparse irrigation in all plant species. In $O$. dictamnus it was positively affected by the soil substrate as well (Table 5).

Table 5. The effect of the main experimental factors, i.e., irrigation frequency, normal ( $\mathrm{n}$ ) or sparse (s) and substrate type (with soil: $3 \mathrm{GC}: 3 \mathrm{P}: 2 \mathrm{~S}: 2 \mathrm{Pu}$ or soilless: $3 \mathrm{GC}: 3 \mathrm{P}: 4 \mathrm{Pu}$ ), and the effect of the experimental treatments, on leaf thickness $(\mu \mathrm{m})$ in July 2012, 2013 or 2014

\begin{tabular}{|c|c|c|c|c|c|}
\hline Main factor ${ }^{2}$ & $\begin{array}{l}\text { C. cneorum } \\
\text { July } 2013\end{array}$ & $\begin{array}{l}\text { O. dictamnus } \\
\text { Sept. } 2012\end{array}$ & $\begin{array}{c}\text { S. athoa } \\
\text { July } 2012\end{array}$ & $\begin{array}{l}\text { A. halimus } \\
\text { July } 2014\end{array}$ & $\begin{array}{l}\text { L. cretica } \\
\text { July } 2014\end{array}$ \\
\hline Normal & $693.2 \pm 10.7 b$ & $350.0 \pm 14.9 b$ & $351.7 \pm 10.5 b$ & $360.0 \pm 7.9 \mathrm{~b}$ & $603.0 \pm 3.9 b$ \\
\hline Sparse & $710.9 \pm 10.7 \mathrm{a}$ & $492.5 \pm 14.9 \mathrm{a}$ & $464.2 \pm 11.2 \mathrm{a}$ & $422.5 \pm 7.9 \mathrm{a}$ & $623.0 \pm 3.9 \mathrm{a}$ \\
\hline Soil & $708.0 \pm 10.7 \mathrm{a}$ & $462.5 \pm 14.9 \mathrm{a}$ & $366.7 \pm 11.2 \mathrm{a}$ & $380.0 \pm 7.9 \mathrm{a}$ & $611.0 \pm 3.9 \mathrm{a}$ \\
\hline Soilless & $696.1 \pm 10.7 \mathrm{a}$ & $380.0 \pm 14.9 b$ & $449.2 \pm 10.5 \mathrm{a}$ & $402.5 \pm 7.9 \mathrm{a}$ & $615.0 \pm 3.9 \mathrm{a}$ \\
\hline Firrig & * & * & * & * & $*$ \\
\hline Fsubst & $n s$ & * & $n s$ & $n s$ & $n s$ \\
\hline Fir $\times \mathrm{s}$ & ns & ns & ns & ns & ns \\
\hline \multicolumn{6}{|l|}{ Treatment $^{y}$} \\
\hline Soil/n & $697.0 \pm 15.2 \mathrm{ab}$ & $315.0 \pm 21.2 \mathrm{c}$ & $350.0 \pm 15.9 \mathrm{~b}$ & $345.0 \pm 11.2 \mathrm{~b}$ & $600.0 \pm 5.6 c$ \\
\hline Soil/s & $719.0 \pm 15.2 \mathrm{a}$ & $610.0 \pm 21.2 \mathrm{a}$ & $383.3 \pm 15.9 b$ & $415.0 \pm 11.2 \mathrm{a}$ & $622.0 \pm 5.6 \mathrm{ab}$ \\
\hline Soilless/n & $689.5 \pm 15.2 \mathrm{~b}$ & $385.0 \pm 21.2 b$ & $353.3 \pm 13.8 \mathrm{~b}$ & $375.0 \pm 11.2 \mathrm{~b}$ & $606.0 \pm 5.6 \mathrm{bc}$ \\
\hline Soilless/s & $702.7 \pm 15.2 \mathrm{ab}$ & $375.0 \pm 21.2 \mathrm{bc}$ & $545.0 \pm 15.9 \mathrm{a}$ & $430.0 \pm 11.2 \mathrm{a}$ & $624.0 \pm 5.6 \mathrm{a}$ \\
\hline
\end{tabular}

${ }^{z}$ Mean $\left( \pm\right.$ SE) comparison in columns within each main factor with Student's $t$ at $P \leq 0.05$; ${ }^{\mathrm{y}} \mathrm{Mean}( \pm \mathrm{SE})$ comparison in columns within treatments with Student's $t$ at $P \leq 0.05$; Means followed by the same letter are not significantly different at $P \leq 0.05 ;{ }^{*}=$ significant at $P \leq 0.05$; ns $=$ not significant

\section{Leaf stomatal resistance $\left(R_{\text {leaf }}\right)$}

In all five plant species, stomatal resistance $\left(R_{\text {leaf }}\right)$ was increased especially in the middle of the summer one d before irrigation in sparsely irrigated plants indicating water limitation. In autumn, its values were normal again, indicating the end of water stress (Figure 1). There was no water limitation observed in any plant species during winter (Figure 3). 
(C1)

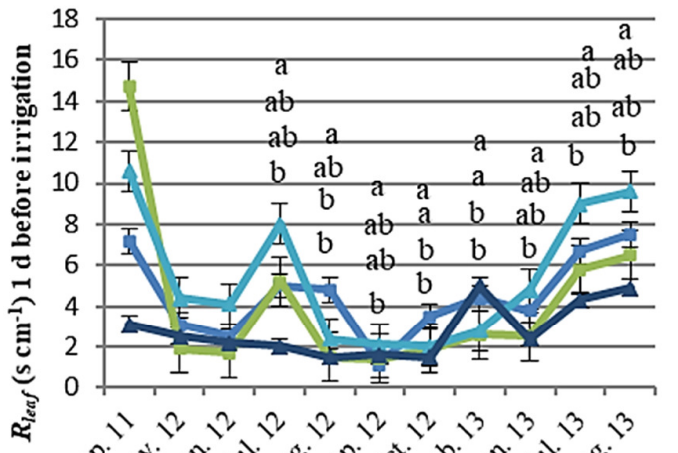

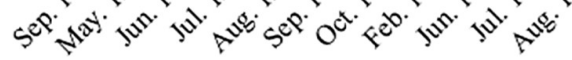

\section{Date (month)

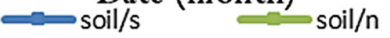 \\ $\Longrightarrow$ s-less/s $\Longrightarrow$ s-less $/ \mathrm{n}$}
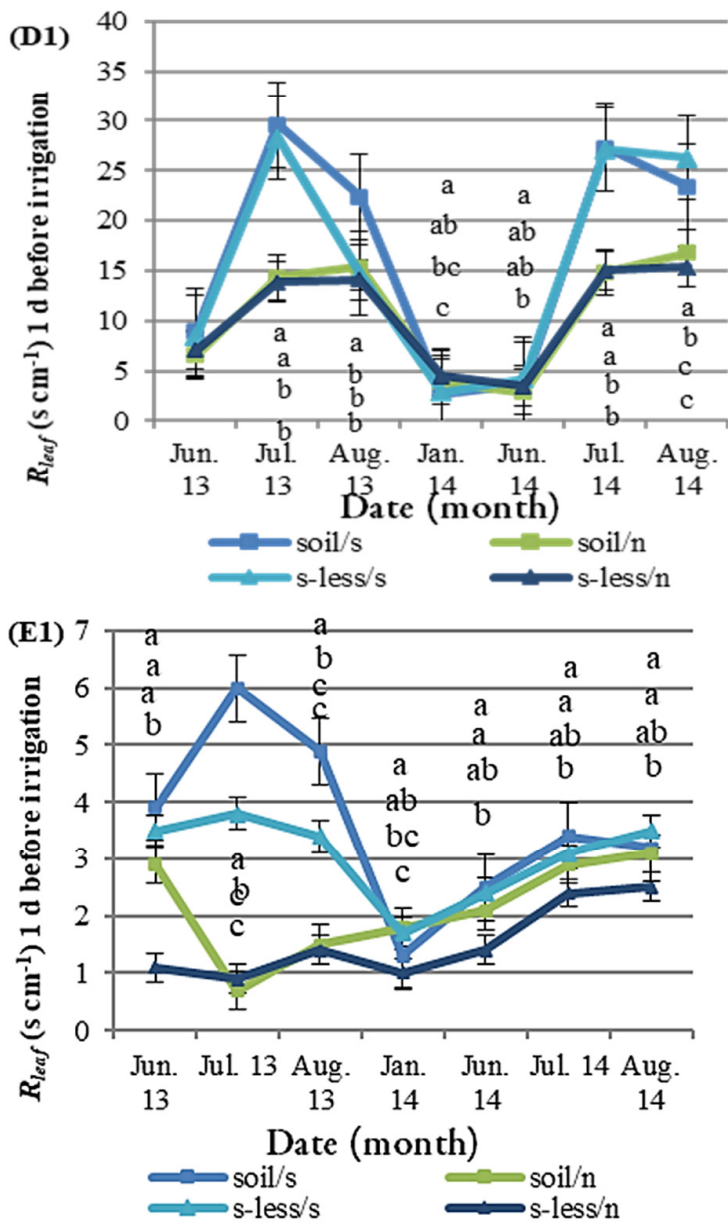

(C2)

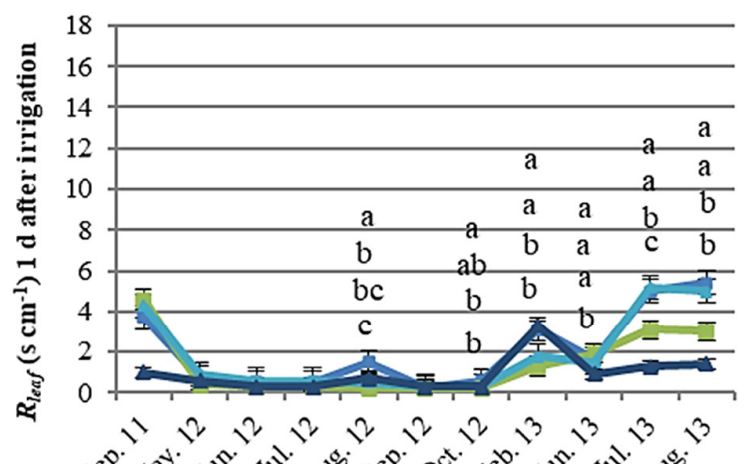

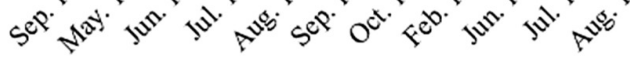
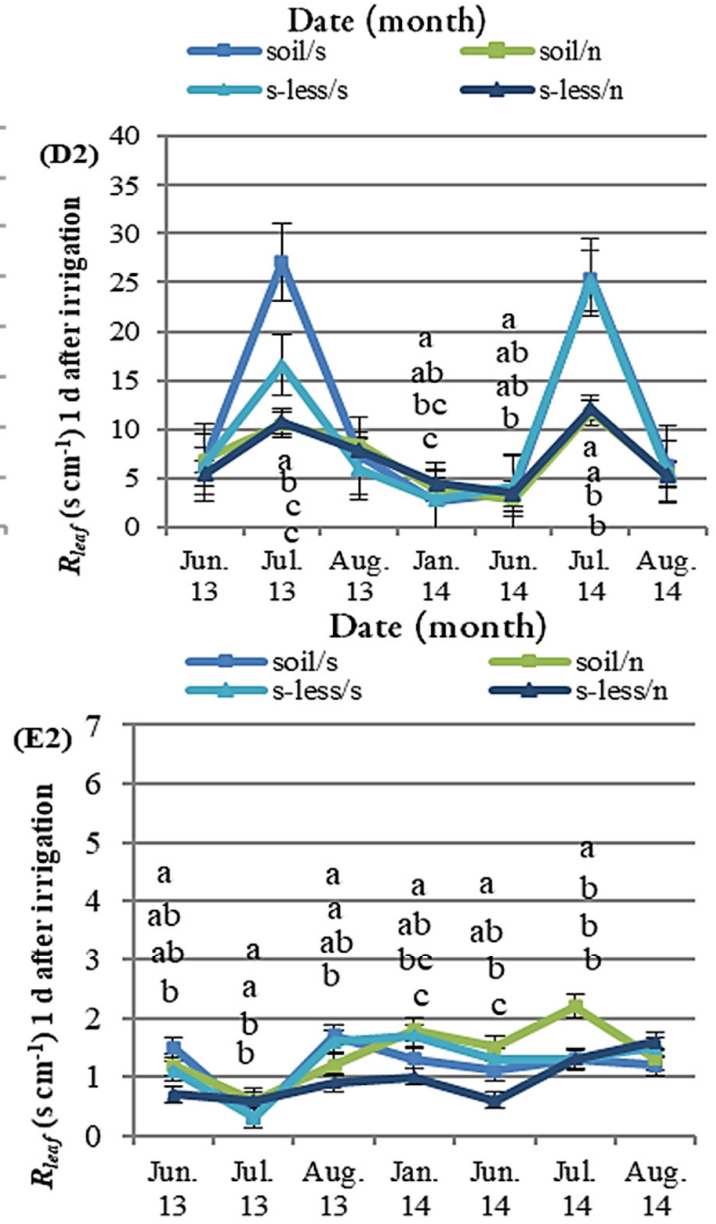

Date (month)

Figure 3. Effect of the experimental treatments that consist of combinations of irrigation frequency $(\mathrm{n}=$ normal or $\mathrm{s}=$ sparse) and substrate type (soil $=3 \mathrm{GC}: 3 \mathrm{P}: 2 \mathrm{~S}: 2 \mathrm{Pu}$ or soilless $=3 \mathrm{GC}: 3 \mathrm{P}: 4 \mathrm{Pu}$ ) on plants' $R_{\text {leaf }}$ $\left(\mathrm{s} \mathrm{cm}^{-1}\right) 1 \mathrm{~d}$ before and $1 \mathrm{~d}$ after an irrigation event during the water stress application periods and once in the winter (Feb. 2013 or Jan. 2014) in (A) C. cneorum, (B) O. dictamnus, (C) S. athoa, (D) A. halimus and (E) L. cretica.

Mean comparison at each date (month) with Fisher's Least Significant Difference (LSD) at $P \leq 0.05$. GC $=$ grape marc compost; $\mathrm{P}=$ perlite; $\mathrm{S}=$ soil; $\mathrm{Pu}=$ pumice 


\section{Maximum quantum yield of PSII photochemistry $\left(\Phi_{P S I O}\right)$}

Generally, after the application of irrigation the maximum quantum yield of PSII ( $\left.\Phi_{\text {PSII }}\right)$ was slightly higher in all treatments. One d before irrigation, the sparsely irrigated plants had the lowest $\Phi_{\text {PSII }}$ values, which never fell to critical levels. This implies that there was no irreversible damage to the photosynthetic apparatus. One $\mathrm{d}$ after an irrigation event, $\Phi_{\text {PSIIo }}$ values completely returned to the normal levels (Figure 4). In winter and spring, the $\Phi_{\mathrm{PSII}}$ in $C$. cneorum, $O$. dictamnus and $S$. athoa there was an indication that it had the lowest values, while in A. halimus and L. cretica the values measured were at the highest range (Figure 4).
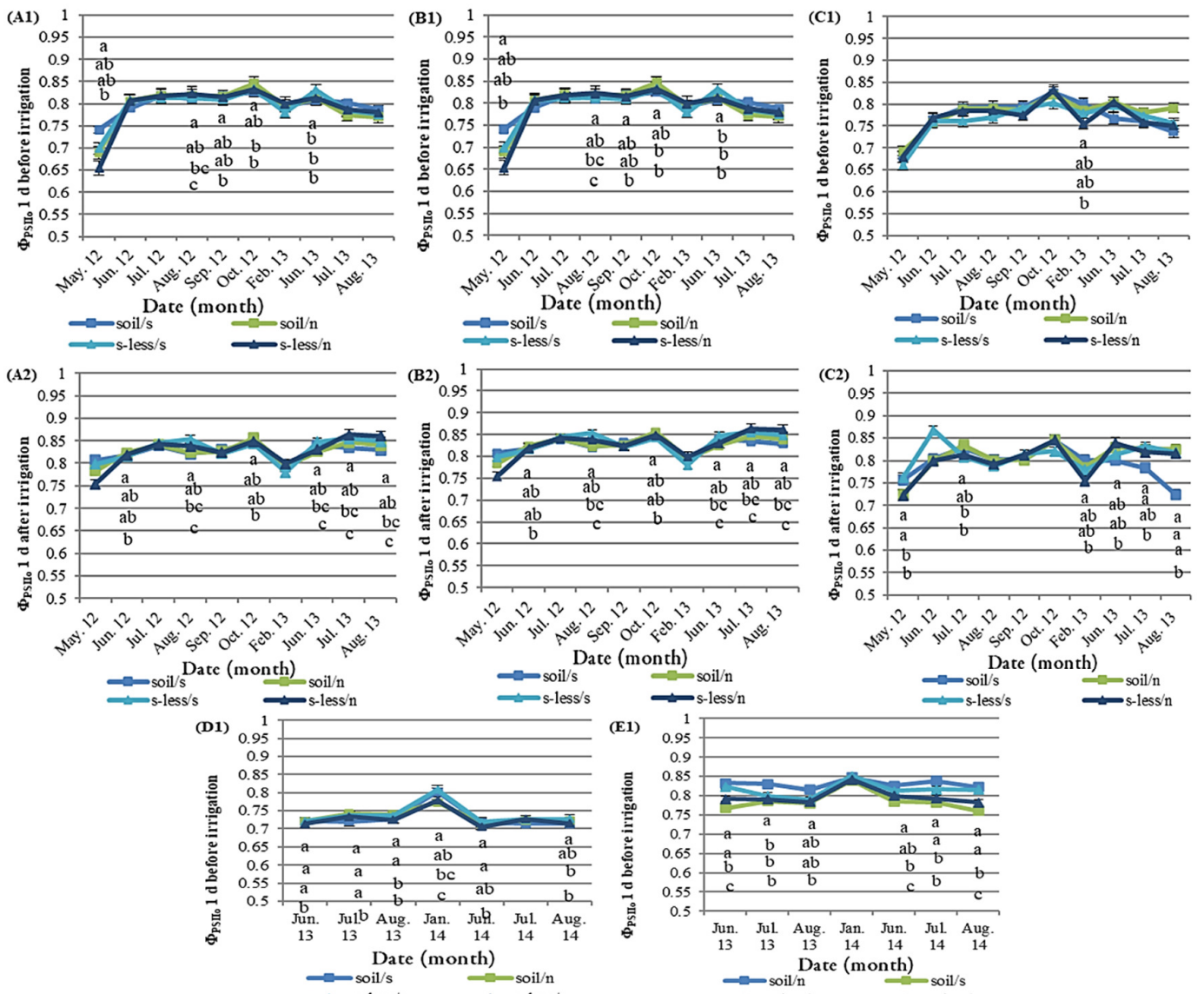
Date (month)

$$
\text { (E1) }
$$
$=$ soil/s $\quad=$ soil/n

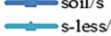

$=$ soil/n
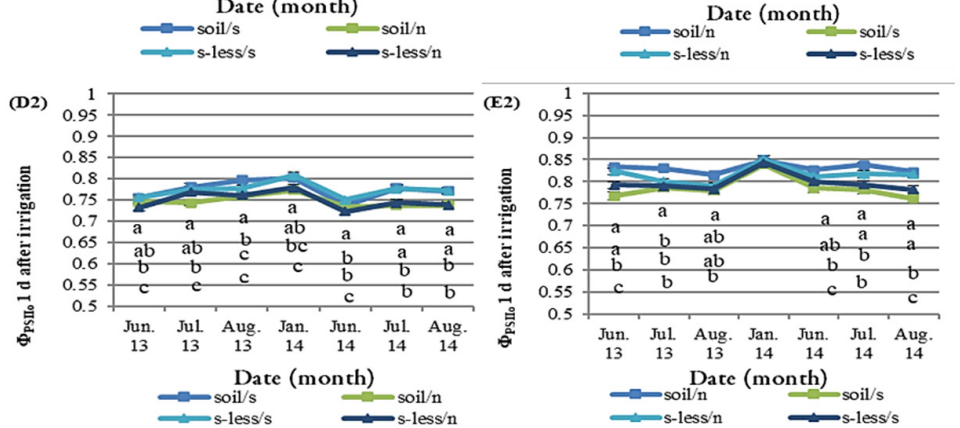

Figure 4. Effect of the experimental treatments that consist of combinations of irrigation frequency $(\mathrm{n}=$ normal or $s=$ sparse) and substrate type (soil = 3GC:3P:2S:2Pu or soilless $=3 \mathrm{GC}: 3 \mathrm{P}: 4 \mathrm{Pu}$ ) on plants' $\Phi_{\text {PSIIo }}$ $1 \mathrm{~d}$ before and $1 \mathrm{~d}$ after an irrigation event during the water stress application periods and once in the winter (Feb. 2013 or Jan. 2014) in (A) C. cneorum, (B) O. dictamnus, (C) S. athoa, (D) A. halimus and (E) L. cretica

Mean comparison at each date (month) with Fisher's Least Significant Difference (LSD) at $P \leq 0.05$. GC $=$ grape marc compost; $\mathrm{P}=$ perlite; $\mathrm{S}=$ soil; $\mathrm{Pu}=$ pumice 


\section{Observation / illustration of leaf blade structure}

The observation of the leaf blade sections in the optical microscope revealed some notable structures. In the plant species C. cneorum (Figure 5A) oblique non-glandular mechanical hairs of particularly long length as well as a carbon cellular inclusion can be seen and in $O$. dictamnus (Figure 5B) irregularly distributed liposomes and hairs are distinguished. In $S$. athoa (Figure 5C) numerous hairs, glands and liposomes appear, while in $A$. halimus (Figure 5D) the "Krantz" type anatomy of the leaf and carbon cellular inclusions in a straight line is evident. In L. cretica (Figure 5E) appear carbon cellular inclusions and solid hairs and in Figure 5F the anatomy of $S$. athoa's glandular hair is clearly visible.

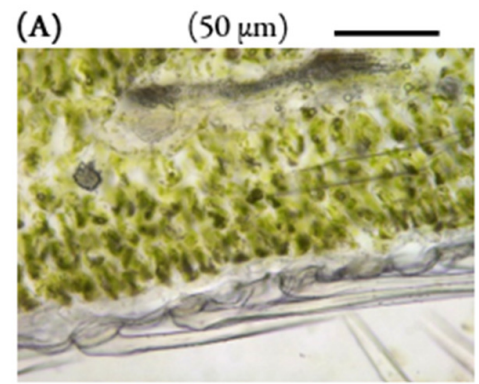

(D)

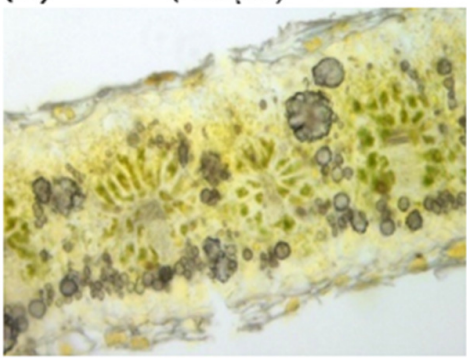

(B)

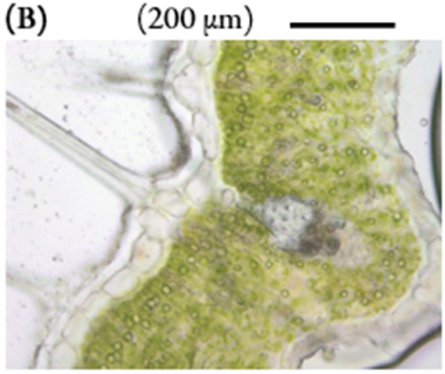

(E) $(200 \mu \mathrm{m})$

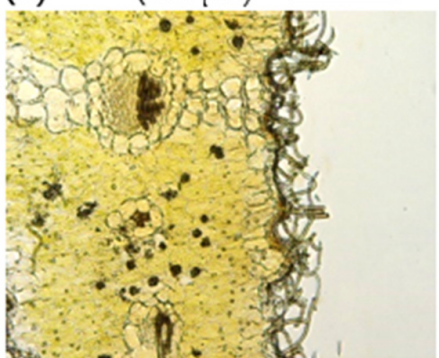

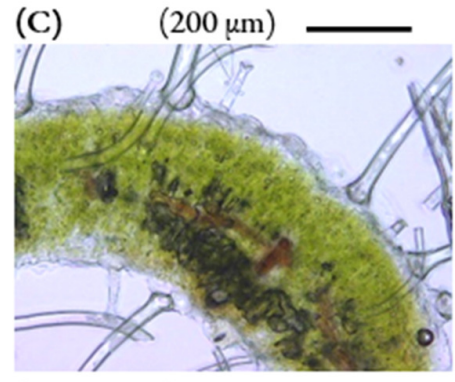

(F)

$(100 \mu \mathrm{m})$

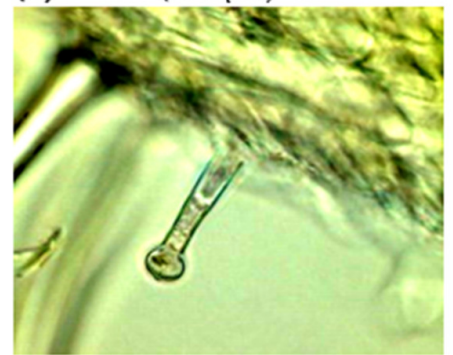

Figure 5. Transverse section of leaf blade in (A) C. cneorum, (B) O. dictamnus, (C) S. athoa, (D) A. halimus, (E) L. cretica and (F) transverse section of $S$. athoa leaf blade, showing the anatomy of the plant hair

\section{Discussion}

\section{Meteorological data}

All periods when irrigation treatments were applied were characterized by high atmospheric temperatures, low atmospheric humidity, as well as almost no rainfall, especially in July and August (Figure 1), a common situation for Greek and in general east-Mediterranean summers. Visual observations of all the plants showed that during the dry season the size of the leaf decreased, in an attempt by the plants to reduce their surface area and thus reduce respiratory water losses. The reduction of the leaf-blade area was reflected in the increase in the leaf-blade thickness (Table 5). This behavior was more pronounced in $O$. dictamnus leaves that appeared after defoliation due to drought, and in $S$. athoa, which sometimes also showed leaf lamina rolling. Therefore, all five species have morphological characters that contribute to a strong limitation of respiratory water losses (Figure 5).

\section{Chemical and physical properties of substrates}

The Electrical conductivity $\left(\mathrm{EC}_{1: 5}\right)$ of the substrates, was initially elevated due to the presence of compost, but over time, a large decrease was observed (Figure 2 A, B) that could be attributed to salt leaching due to autumn and winter rainfalls, in agreement with previous studies (Papafotiou et al., 2005; 2013). Thus, 
at the end of the first series of experiments (C. cneorum, $O$. dictamnus and $S$. athoa), the $\mathrm{EC}_{1: 5}$ of the soilless substrate was similar to that of the soil substrate (Figure 3A). The substrates had a similar $\mathrm{pH}$ at planting and at the end of the experiment (Figure 2A, B), thus possibly excluding a different nutrient environment in plants of the two substrate types. Grape marc compost had adequate quantities of nutrients (Tassoula et al., 2015) to support growth of all five plant species during the experimental period. The two substrates had similar water retention curves with the difference that the substrate with soil held more water at saturation and at low suctions $(0-40 \mathrm{~cm})$ (Tassoula et al., 2015).

The saturated weights of the both substrates (Tassoula et al., 2015) are lower than those considered acceptable for extensive green roofs (Dunnett and Kingsbury, 2008).

\section{Plant growth}

Establishment and growth of all plant species in the green roof were successful under all experimental treatments. All five species survived the harsh conditions of winter as well as the summer drought period. Although there were periods of growth inhibition, which differed little from species to species, at the end of the experiments a satisfactory degree of plant growth (Tables 1,2) and an excellent appearance of the green roof was observed, as the plants were overlapping the experimental modules. In two out of the five species, i.e., C. cneorum and $A$. halimus, canopy diameter and dry weight was promoted by soil amended substrate, in three out of the five species, i.e., O. dictamnus, A. halimus and L. cretica these growth parameters were promoted by normal irrigation, while the combination of soil substrate with normal irrigation strongly promoted growth in $O$. dictamnus and $A$. halimus (Tables 1,2). Soil's beneficial effect on growth may be due to soil ability to retain more moisture (Nektarios et al., 2011; Tassoula et al., 2015). In an experiment concerning Thymus citriodorus using the same substrates, plants' horizontal diameter and dry weight of canopy appeared to be positively affected by the presence of soil in the substrate, regardless of irrigation (Papafotiou and Adami, 2020). However, due to the fact that natural soils are heavy for use in green roofs, researchers are experimenting to develop light substrates (Beattie and Berghage, 2004; Papafotiou et al., 2018; Eksi and Rowe, 2019). Among the co-cultivated species $C$. cneorum, $O$. dictamnus and $S$. athoa, the latter two developed a similar horizontal diameter, while the highest diameter, about twice in most treatments, was presented by $C$. cneorum (Table 1 ). However, $C$. cneorum in most treatments showed a similar or lower dry weight to $S$. athoa, and $O$. dictamnus showed the lowest dry weight compared to all species (Table 2). The high dry weight of $S$. athoa was probably due to its mostly woody shoots, compared to the other two species. Among the co-cultivated $A$. halimus and $L$. cretica, the former had the highest diameter and dry weight, being a fast-growing bush, in contrast to L. cretica that is a small rather slowly growing shrub. Sparse irrigation adversely affected plant diameter and dry weight in $O$. dictamnus, $A$. halimus and $L$. cretica but the plants had satisfactory canopy growth (Tables 1,2 ) considering that in green roofs the maximum growth is not intended. Throughout the experimental period, regardless of season, a large part of the lower leaves that came in contact with the substrate dried and fell off, while there was a constant growth of new foliage. The reduced growth of some of the plants in the present study under sparse irrigation, without being a negative element for the green roof, is a common effect of water stress on plant growth (Flexas et al., 2002; Singh et al., 2019).

A key factor for the growth of all five plant species could be the use of grape marc compost containing nutrients in adequate quantities for the growth of these species. Its high content of $\mathrm{K}$ could be a reason they coped well with the harsh green roof s conditions (Cakmak, 2005; Papafotiou et al., 2013). In the present study, the decomposition of the compost was very slow and no reduction in the depth of the substrate was observed during the two-year experimental period. In combination with the partial change of the foliage, which constantly enriched the substrate with organic matter (Moran et al., 2005), led to no problem in plant growth.

The high percentage of pumice participation in the growth substrates, probably favored plant growth, as well, as researchers recommend it as a substrate material for extensive green roofs, at a rate of $45 \%$ (Eksi and Rowe, 2019), and even at a rate of $70 \%$ (Fassman and Simckock, 2012). Pumice at participation rates of 20\%, 
$30 \%$ and $40 \%$ was also considered suitable for growing turfgrass (Nydrioti et al., 2009; Ntoulas et al., 2012), as well as the xerophyte plant Dianthus fruticosus (Nektarios et al., 2011).

\section{Water use efficiency}

Plant species resistant to deficient irrigation are able to maintain WUE, preventing water loss in various ways (Yu et al., 2020). Otherwise WUE decreases alarmingly, leading to reduced plant biomass (Farooq et al., 2009). In A. halimus and L. cretica WUE was higher under sparse irrigation compared to normal irrigation (Table 3). Similarly, in a cotton plantation half the amount of irrigation resulted in higher WUE values than in full irrigation (Howell et al., 2004). A. halimus' higher WUE values compared to all other species were probably due to its $\mathrm{C} 4$ photosynthesis pathway versus $\mathrm{C} 3$ of the other species (Ghannoum et al., 2011). In the other three species, $C$. cneorum, $O$. dictamnus and $S$. athoa, WUE was not affected by irrigation frequency (Table 3). C. cneorum was the only species that its WUE was affected by the type of substrate, being higher in the soil amended one (Table 3). In C. cneorum, $O$. dictamnus and $S$. athoa the combination of soil substrate with normal irrigation lead to WUE values similar to the combination of soilless substrate and sparse irrigation, while in A. halimus and L. cretica the later combination presented higher values of WUE. This is an important result as it shows that a lightweight substrate combined with reduced water supply secures high WUE in an urban green roof when the appropriate plant species are incorporated. According to Bresta et al. (2011) WUE is improving while water stress is increasing, up to a threshold. High WUE values were presented by $A$. halimus (Table 3) and this was expected as it is a C4 plant that easily adapts to dry climates (Le Houerou, 1992). Something also expected were the low WUE values that were presented by L.cretica for its irrigation program contained sparse and regular irrigation applications with the shortest interval compared to the other plant species. International standards for green roofs' irrigation, such as the German F.L.L. (2010), refer to the climatic conditions prevailing in the northern countries. Therefore, many researchers argue that when green roofs are located in arid areas, irrigation programs should be implemented depending on the conditions, especially during summer (William et al., 2016; Getter and Rowe, 2006). In environments where the thermal island effect is enhanced, even sparse irrigation may be able to contribute to its reduction (Dunnett and Kingsbury, 2010). Therefore, in cases where there is the possibility of irrigation, it is important to take into account the above environmental parameter.

\section{Total chlorophyll concentration (Chl tor)}

In most plant species, the sparsely irrigated plants and those cultured on soilless substrate showed lower $\mathrm{Chl}_{\text {tot }}$ values compared to those under normal irrigation and soil substrate during the hot dry season (Table 4), indicating water stress (Gheek Batra et al., 2014).

\section{Leaf thickness}

Sparse irrigation caused the increase of leaf thickness during the summer period in all plant species (Table 5). Increasing the thickness of the leaves under water stress is a xerophytic characteristic that might permit relatively efficient photosynthesis with rationalized water losses (Turner, 1994). The range of the difference of the leaf thickness between sparse and normal irrigation frequency in plant species was $68.8 \mu \mathrm{m}$.

\section{Leaf stomatal resistance $\left(R_{\text {leaf }}\right)$}

$\mathrm{R}_{\text {leaf }}$ depends on the plant species and is proportional to the stage of development and directly related to climatic factors and water availability. One $\mathrm{d}$ before an irrigation event, the highest $\mathrm{R}_{\text {leat }}$ values in all five species were caused by treatments involving sparse irrigation, regardless of the type of substrate, and one $\mathrm{d}$ after an irrigation event the $\mathrm{R}_{\text {leaf }}$ values decreased significantly in all species, thus confirming that plants under sparse irrigation were water stressed (Figure 3). It has been shown previously the ability of many plant species to adapt normally to arid conditions and to return to normal within 48 hours after re-irrigation (Souza et al., 2010). A. halimus recorded high $\mathrm{R}_{\text {leaf }}$ values both before and after irrigation (Figure 3), which was to be expected since it 
had the longest period between two irrigation events and a large above ground growth. For a number of xerophytic aromatic and medicinal plants, as Ballota acetabulosa, Helichrysum orientale, Melissa officinalis, Rosmarinus officinalis and Salvia fruticosa, has been found to have the highest $\mathrm{R}_{\text {leaf }}$ values when irrigated sparingly (Kokkinou et al., 2016). Many annual or perennial species that appear capable of growing under water scarcity follow a water-saving strategy, also referred to as "drought avoidance". According to this strategy, plants minimize loss and maximize uptake of water. Functional acclimation, such as stricter stomatal closure, and anatomical characteristics such as thick cell walls of epidermal cells, thick cuticular layer, glandular exudation of resinous mixtures or pubescence, further restrict transpiration by increasing resistance to water vapor diffusion and minimizing thermal energy input by reflecting more light (Chaves et al., 2003; Karabourniotis et al., 2020a). For example, an important anatomical feature in $A$. halimus is the glandular hairs on the surface of its leaves (Mozafar and Goodin, 1970), which are superficially covered by a waxy material and are attached to epidermal cells (Smaoui et al., 2011). In fact, all plant species in the present research, namely $C$. cneorum (Figure 5A), O. dictamnus (Figure 5B), S. athoa (Figure 5C-F), A. halimus (Figure 5D) and L. cretica (Figure $5 \mathrm{E})$, appear intensely sericeous in their lamina, having short and sometimes longer hairs. Non-glandular hairs, as a kind of mechanical, optical and chemical barrier, protect the leaves from insects or pathogens, as well as from ultraviolet radiation that increases leaf temperature (Karabourniotis and Fasseas, 1996; Stavrianakou et al., 2010; Karabourniotis, 2020a). The role of essential oils in plants is to help them interact with the environment, while protecting them from herbivores and pathogens (Hallahan, 2000). In S. athoa (Figure 5CF), the peltate glands are made up of 8-12 secretory cells, the density of which varies from season to season. These play a protective role against excess sunlight, judged by the observation that they increase after winter (Bozambalidis, 2008; Figueiredo et al., 2008). In addition, C. cneorum (Figure 5A), A. halimus (Figure 5D), and $L$. cretica (Figure 5E), contain carbon cellular inclusions in their leaves, which may play a protective role (Karabourniotis et al., 2020b). Researchers claim that leaf crystals have various roles, such as storage and regulation of calcium cations (Franceschi and Horner, 1980), plant protection (Ward et al., 1997), inactivation of heavy metals (Zindler-Frank, 1991), osmoregulation (Raven and Smith, 1976), tissue support, etc. In addition, Tooulakou et al. (2016) demonstrated that the calcium oxalate crystals in the leaves could function as a biochemical reservoir that collects non-atmospheric carbon, especially during the night, and during the $\mathrm{d}$, crystal degradation provides additional carbon for photosynthetic assimilation, especially in drought conditions. This new photosynthetic pathway called "alarm photosynthesis" seems to provide a number of adaptive benefits, such as water economy, reduction of carbon losses in the atmosphere and protection of photosystem II, roles that justify the presence of carbon cellular inclusions in plants (Karabourniotis et al., 2020b).

\section{Maximum quantum yield of PSII photochemistry $\left(\Phi_{\text {PSIIo }}\right)$}

According to the PSII photochemical parameters, one $\mathrm{d}$ before an irrigation event, when the plants were facing the maximum water limitation as $\mathrm{R}_{\text {leaf }}$ values revealed (Figure 3), photochemical efficiency of PSII photochemistry $\left(\Phi_{\mathrm{PSII}}\right)$ values never fell to critical levels, ruling out irreversible damage to the photosynthetic machinery (Figure 4). Both $\Phi_{\text {PSIIo }}$ and $\mathrm{R}_{\text {leaf }}$ show significant response to the increase of water availability as they change notably after each irrigation event compared to the $\mathrm{d}$ before. So, they show promising to be used as sensitive water stress probes in similar applications. This conclusion is further supported by the observation that the $\mathrm{d}$ after every irrigation event, $\Phi_{\text {PSII }}$ values were fully recovered (Figure 4). It has been shown in the past that plants subjected to water stress may recover after irrigation, as in the case of Eucalyptus globulus (Correia et al., 2013). In addition, a drought-tolerant chrysanthemum variety had better photosynthesis during water stress than a drought-sensitive one (Sun et al., 2013). On the other hand, the water stress brought about a significant reduction of $\Phi_{\text {PSIIo }}$ in the succulent Sedum alba (Rowe et al., 2014). Limiting factors for photosynthesis during water stress vary depending on the plant species (Galmes et al., 2007), the intensity of previous water stresses (Flexas et al., 2009), light and temperature (Galle et al., 2009), plant age (Varone et al., 2012 ) and the application of successive stress/recovery cycles (Galle et al., 2011). Values of $\Phi_{\text {PSIIo }}$ indicating 
healthy plants are within an empirical range of 0.78-0.84 (Maxwell and Johnson, 2000). In none of the five plant species did the $\Phi_{\text {PSIIo }}$ value fall below 0.70 , indicating that PSII was affected albeit not irreversibly. $\Phi_{\text {PSIIo }}$ was at its lowest levels in May, possibly because the plants emerged from a long period without adequate rain events (Figure 2) and no-irrigation. In winter also, the $\Phi_{\text {PSII }}$ in $C$. cneorum, $O$. dictamnus and $S$. athoa had the lowest values, while in $A$. halimus and $L$. cretica the values measured were at the highest range (Figure 4 ). This could be due to the prevailing temperatures that were lower in February $2013\left(T_{\max } 13,2, T_{\min } 5,7, T_{\text {average }} 9,3\right)$ compared to January $2014\left(\mathrm{~T}_{\max } 17,4, \mathrm{~T}_{\min } 9,4, \mathrm{~T}_{\text {average }} 13,4\right)$.

A decisive factor for the results of the research was the mat for moisture retention and protection of insulation, especially during periods of water stress, which probably improved irrigation efficiency as shown by the results of other experiments (Rowe et al., 2014). It is very likely that when the roots of the plants were elongated, they were pumping water from the infrastructure and therefore the moisture of the substrate did not affect the growth of the plants, which confirms the positives of the infrastructure especially during the period of water stress (Savi et al., 2013).

\section{Conclusions}

Extensive type green roofs are very important for arid and semi-arid regions such as Eastern Mediterranean, especially if applied on a large scale in urban environment and join green networks in order to improve the microclimate (Getter and Rowe, 2006). More research and experimental data are needed to determine as many parameters as possible for substrates and plant species that can survive in the xerothermic urban conditions, with minimal irrigation. The Mediterranean xerophytes $C$. cneorum L., $O$. dictamnus L., $S$. athoa L., A. halimus L. and L. cretica L. showed satisfactory growth, while cultivated under sparse irrigation and on soilless (light) substrate. By monitoring their particular moisture requirements and adjusting the duration between irrigations, all five are suitable plant species for use on extensive green roofs in the Mediterranean region and other areas with similar to the Mediterranean climate.

\section{Authors' Contributions}

Conceptualization: MP and LT; Data curation: LT; Formal analysis: LT; Investigation: LT and MP; Methodology: MP, GK and GL; Resources: MP, GK and GL; Supervision: MP; Writing - original draft: LT and MP. All authors read and approved the final manuscript.

\section{Acknowledgements}

This research received no specific grant from any funding agency in the public, commercial, or not-forprofit sectors.

\section{Conflict of Interests}

The authors declare that there are no conflicts of interest related to this article. 


\section{References}

Abass F, Ismail LH, Wahab IA, Elgadi AA (2020). A review of green roof: definition, history, evolution and functions. IOP Conference Series: Materials Science and Engineering 713:012048. https://doi.org/10.1088/1757$899 X / 713 / 1 / 012048$

Aligiannis N, Kalpoutzakis E, Chinou IB, Mitakou S, Gikas E, Tsarbopoulos A (2001). Composition and antimicrobial activity of the essential oils of five taxa of Sideritis from Greece. Journal of Agriculture and Food Chemistry 49(2):81-815. https://doi.org/10.1021/jf001018w

Andric I, Kamal A, Al-Ghamdi SG (2020). Efficiency of green roofs and green walls as climate change mitigation measures in extremely hot and dry climate: Case study of Qatar. Energy Reports 6:2476-2489. https://doi.org/10.1016/j.egyr.2020.09.006

Beattie DJ, Berghage RD (2004). Design criteria for a green roof medium Proc. 2nd Intl. Green Roof Conf.: Greening Rooftops for Sustainable Communities 2:305-317. https://www.osti.gov/etdeweb/biblio/20861876

Benvenuti S (2014). Wildflower green roofs for urban landscaping, ecological sustainability and biodiversity. Landscape and Urban Planning 124:151-161. https://doi.org/10.1016/j.landurbplan.2014.01.004

Bosabalidis AM (2002). Structural features of Origanum. In: Kintzios S (Ed). Oregano: The Genera Origanum and Lippia. CRC Press, London https://books.google.gr

Bresta P, Nikolopoulos D, Economou G, Vahamidis P, Lyra D, Karamanos A, Karabourniotis G (2011). Modification of water entry (xylem vessels) and water exit (stomata) orchestrates long term drought acclimation of wheat leaves. Plant Soil 347:179-193. https://doi.org/10.1007/s11104-011-0837-4

Cakmak I (2005). The role of potassium in alleviating detrimental effects of abiotic stresses in plants. Journal of Plant Nutrition and Soil Science 168:521-530. https://doi.org/10.1002/jpln.200420485

Cameron RWF, Blanusa T, Taylor JE, Salisbury A, Halstead AJ, Henricot B, Thompson K (2012). Urban Forestry and Urban Greening 11(2):129-137. https://doi.org/10.1016/j.ufug.2012.01.002

Chaves MM, Maroco J, Pereira JS (2003). Understanding plant responses to drought-from genes to the whole plant. Functional Plant Biology 30:239-264. https://www.researchgate.net

Cook-Patton SC, Bauerle TL (2012). Potential benefits of plant diversity on vegetated roofs: a literature review. Journal of Environmental Management 106:85-92. https://doi.org/10.1016/j.jenvman.2012.04.003

Correia B, Pinto-Marijuanb M, Neves L, Brossac R, Celeste-Dias M, Costa A, ...Pinto G (2013). Water stress and recovery in the performance of two Eucalyptus globulus clones: physiological and biochemical profiles. Physiologia Plantarum 150: 580-592. https://doi.org/10.1111/ppl.12110

Cristiano E, Deidda R, Viola F (2021). The role of green roofs in urban water-energy-food-ecosystem nexus: A review. Science of the Total Environment 756:143876. https://doi.org/10.1016/j.scitotenv.2020.143876

Dai A (2013). Increasing drought under global warming in observations and models. Nature Climate Change 3:52-58. https://doi:10.1038/nclimate1633

Dunnett NP, Kingsbury N (2008). Planting green roofs and living walls. Timber Press (2nd ed), Portland, Oregon.

Eksi M, Rowe DB (2019). Effect of substrate depth and type on plant growth for extensive green roofs in a Mediterranean climate. Journal of Green Building 14(2):29-44. https://doi.org/10.3992/1943-4618.14.2.29

Fan Y, Wang C, Nan Z (2018). Determining water use efficiency of wheat and cotton: a meta-regression analysis. Agricultural Water Management 199,48-60. https://ideas.repec.org

Farooq M, Wahid A, Kobayashi N, Fujita D, Basra SMA (2009). Plant drought stress: Effects, mechanisms and Management. Sustainable Agriculture 153-188. https://hal.archives-ouvertes.fr/hal-00886451/document

Fassman E, Simcock A (2012). Moisture measurements as performance criteria for extensive living roof substrates. Environmental Engineering 138(8):841-851. https://doi.org/10.1061/(ASCE)EE.1943-7870.0000532

Feitosa RC, Wilkinson SJ (2020). Small-scale experiments of seasonal heat stress attenuation through a combination of green roof and green walls. Journal of Cleaner Production 250:119443. https://doi.org/10.1016/j.jclepro.2019.119443

Figueiredo AC, Barroso JG, Pedro LG, Scheffer JC (2008). Factors affecting secondary metabolite production in plants: volatile components and essential oils. Flavour and Fragrance Journal 23(4):213-226. https://doi.org/10.1002/ffj.1875

Flexas J, Barón M, Bota J, Ducruet JM, Gallé A, Galmés J ... Medrano H (2009). Photosynthesis limitations during water stress acclimation and recovery in the drought-adapted Vitis hybrid Richter-110 (V. berlandieri $\times$ V. rupestris). Journal of Experimental Botany 60:2361-2377. https://doi.org/10.1093/jxb/erp069 
Flexas J, Medrano H (2002). Drought-inhibition of photosynthesis in $\mathrm{C}_{3}$ plants: stomatal and non-stomatal limitation revisited. Annals of Botany 89:183-189. https://doi.org/10.1093/aob/mcf027

FLL (2010). Guideline for the planning, execution and upkeep of green-roof sites. (English ed.). Forschungsgesellschaft Landschaftsentwicklung Landschaftsbau.

Fokialakis N, Kalpoutzakis E, Tekwani BL (2006). Evaluation of the antimalarial and antileishmanial activity of plants from the Greek island of Crete. Journal of Natural Medicines 61(1):38. https://doi.org/10.1007/s11418-006$0013-y$

Franceschi VR, Horner HT (1980). Calcium oxalate crystals in plants. The Botanical Review 46:360-427. https://doi.org/10.1007/BF02860532

Galle A, Florez-Sarasa I, Aououad H, Flexas J (2011). The Mediterranean evergreen Quercus ilex and the semi-deciduous Cistus albidus differ in their leaf gas exchange regulation and acclimation to repeated drought and re-watering cycles. Journal of Experimental Botany 62:5207-5216. https://doi.org/10.1007/s11418-006-0013-y

Galle A, Florez-Sarasa I, Tomas M, Pou A, Medrano H, Ribas-Carbo M, Flexas J (2009). The role of mesophyll conductance during water stress and recovery in tobacco (Nicotiana sylvestris): acclimation or limitation? Journal of Experimental Botany 60:2379-2390. https://doi.org/10.1093/jxb/erp071

Galmés J, Medrano H, Flexas J (2007). Photosynthetic limitations in response to water stress and recovery in Mediterranean plants with different growth forms New Phytologist 175:81-93. https://doi.org/10.1093/jxb/erp071

Getter K, Rowe B (2006). The role of extensive roofs in sustainable development. Hortsciensce 41(5):1276-1285. http://hortsciashspublications.org

Ghannoum O, Evans JR, Von Caemmerer S (2011). C4 photosynthesis and related $\mathrm{CO}_{2}$ concentrating mechanisms. Chapter 8. Springer, Dordrecht pp 129-146. https://www.researchgate.net

Gheek Batra N, Sharma V, Kumari N (2014). Drought-induced changes in chlorophyll fluorescence, photosynthetic pigments, and thylakoid membrane proteins of Vigna radiate. Journal of Plant Interactions 9(1):712-721. https://doi.org/10.1080/17429145.2014.905801

Geerts S, Raes D (2009). Deficit irrigation as an on-farm strategy to maximize crop water productivity in dry areas. Agricultural Water Management 96(9):1275-1284. https://doi.org/10.1016/j.agwat.2009.04.009

González-Burgos E, Carretero ME, Gómez-Serranillos MP (2011). Sideritis spp.: Uses, chemical composition and pharmacological activities-A review. Journal of Ethnopharmacology 135(2):209-225. https://doi.org/10.1016/j.jep. 2011.03.014

Hallahan DL (2000). Monoterpenoid biosynthesis in glandular trichomes of labiate plants. Advances in Botanical Research 31:77-120. https://doi.org/10.1016/S0065-2296(00)31007-2

Hatfield JL, Dold C (2019). Water-Use Efficiency: Advances and challenges in a changing climate. Frontiers in Plant Science 10:103. https://doi.org/10.3389/fpls.2019.00103

IPCC (2014). Climate change: synthesis report. In: Pachauri RK, Meyer LA (Eds). Contribution of Working Groups I, II and III to the Fifth Assessment Report of the Intergovernmental Panel on Climate Change. Geneva, Switzerland, pp 151.

IPCC (2007). Climate change: 2007 synthesis report (Contribution of Working Groups I, II and III to the Fourth Assessment Report of the Intergovernmental Panel on Climate Change). Geneva - IPCC.

Jusselme MD, Pruvost C, Motard E, Giusti-Miller S, Frechault S, Alphonse V, ... Mora P (2019). Increasing the ability of a green roof to provide ecosystem services by adding organic matter and earthworms. Applied Soil Ecology 143:61-69. https://doi.org/10.1016/j.apsoil.2019.05.028

Karabourniotis G, Liakopoulos G, Nikolopoulos D, Bresta P (2020a). Protective and defensive roles of non-glandular trichomes against multiple stresses: structure-function coordination. Journal of Forestry Research 31:1-12. https://doi.org/10.1007/s11676-019-01034-4

Karabourniotis G, Horner HT, Bresta P, Nikolopoulos D, Liakopoulos G (2020b). New insights into the functions of carbon-calcium inclusions in plants. New Phytologist. https://doi.org/10.1111/nph.16763

Karabourniotis G Fasseas K (1996). The dense indumentum with its polyphenol content may replace the protective role of the epidermis in some young xeromorphic leaves. Canadian Journal of Botany 74(3):347-351. https://doi.org/10.1139/b96-043

Katerji N, M Mastrorilli G Rana (2008). Water use efficiency of crops cultivated in the Mediterranean region: Review and analysis. European Journal of Agronomy 28(4):493-507. https://doi.org/10.1016/j.eja.2007.12.003 
Tassoula L et al. (2021). Not Bot Horti Agrobo 49(2):12283

Kokkinou I, Ntoulas N, Nektarios PA, Varela D (2016). Response of native aromatic and medicinal plant species to water stress on adaptive green roof systems. HortScience 51(5):608-614. https://doi.org/10.21273/HORTSCI.51.5.608

Kondo MC, Fluehr JM, McKeon T, Branas CC (2018). Urban green space and its impact on human health. International Journal of Environmental Research and Public Health 15(3):445. https://doi.org/10.3390/ijerph15030445

Langemeyer J, Wedgwood D, McPhearson T, Baro F, Madsen AL, Barton DN (2020). Creating urban green infrastructure where it is needed - A spatial ecosystem service-based decision analysis of green roofs in Barcelona. Science of the Total Environment 707:135487. https://doi.org/10.1016/j.scitotenv.2019.135487

Le Houérou HH (1992). The role of saltbushes (Atriplex spp.) in arid land rehabilitation in the Mediterranean Basin: a review. Agroforestry Systems 18:107-148. https://doi.org/10.1007/BF00115408

Lichtenthaler HK (1987). Chlorophylls and carotenoids: pigments of photosynthetic biomembranes. Methods in Enzymology 148:350-1000. https://doi.org/10.1016/0076-6879(87)48036-1

Liolios CC, Gortzi O, Lalas S, Tsaknis J, Chinou I (2009). Liposomal incorporation of carvacrol and thymol isolated from the essential oil of Origanum dictamnus L. and in vitro antimicrobial activity. Food Chemistry 112(1):77-83. https://Www.sciencedirect.com

Liu W, Engel BA, Chen W, Wei W, Wang Y, Feng Q (2021). Quantifying the contributions of structural factors on runoff water quality from green roofs and optimizing assembled combinations using Taguchi method. Journal of Hydrology 593:125864. https://doi.org/10.1016/j.jhydrol.2020.125864

Loder A (2014). There's a meadow outside my workplace: A phenomenological exploration of aesthetics and green roofs in Chicago and Torondo. Landscape and Urban Planning 126:94-106. https://doi.org/10.1016/j.landurbplan.2014.01.008

Lundholm JT, Peck SW (2008). Frontiers of green roof ecology. Urban Ecosystems 11:335-337. https://doi.org/10.1007/s11252-008-0070-y

Martinetti L, Tosca A, Spoleto P, Valaguss M, Gatt A (2018). Evaluation of water stress tolerance of some species suitable for extensive green roofs. Acta Horticulturae 1215:113-116. https://doi.org/10.17660/ActaHortic.2018.1215.20

Maxwell K, Johnson GN (2000). Chlorophyll fluorescence-a practical guide. Journal of Experimental Botany 51:659-668. https://doi.org/10.1093/jexbot/51.345.659

Moore TL, Rodak CM, Vogel JR (2017). Urban stormwater characterization, control and treatment. Water Environment Research 89(10):1876-1927. https://doi.org/10.2175/106143017X15023776270692

Moran A, Hunt W, Smith J (2005). Hydrologic and water quality performance from green roofs in Goldsboro and Raleigh, North Carolina. Greening Rooftops for Sustainable Communities, Proceedings of the Third North American Green Roofs 2005 Conference, The Cardinal Group, Washington, DC Toronto.

Mozafar A, Goodin JR (1970). Vesiculated hairs: a mechanism for salt tolerance in Atriplex halimus L. Plant Physiology 45:62-65. https://doi.org/10.1104/pp.45.1.62

Nektarios PA, Ntoulas N, McElroy S, Volterrani M, Arbis G (2011). Effect of olive mill compost on native soil characteristics and tall fescue turfgrass development. Agronomy Journal 103(5):1524. https://doi.org/10.2134/ agronj2011.0145.

Nila MUS, Beierkuhnlein C, Jaeschke A, Hoffmann S, Hossain ML (2019). Predicting the effectiveness of protected areas of Natura 2000 under climate change. Ecological Processes 8(1):13. https://doi.org/10.1186/s13717-019-01686

Ntoulas N, Nektarios PA, Spaneas K, Kadoglou N (2012). Semi-extensive green roof substrate type and depth effects on Zoysia matrella 'Zeon" growth and drought tolerance under different irrigation regimes. Acta Agriculturae Scandinavica, Section B-Plant Soil Science 62(1). https://www.tandfonline.com/doi/abs/10. 1080/09064710.2012.681391

Nydrioti E, Papadopoulou M, Nektarios PA (2009). Turfgrass growth and evapotranspiration in intensive green roof systems. Acta Horticulturae 881:721-724. https://doi.org/10.17660/ActaHortic.2010.881.118

Palazón J, Moyano E, Bonfill M, Cusidó RM, Piñol MT (2006). Tropane alkaloids in plants and genetic engineering of their biosynthesis. Floriculture, Ornamental and Plant Biotechnology, Volume II, Global Science Books, UK pp 219.

Papafotiou M, Adami I (2020). Effect of substrate type and irrigation frequency on growth of the bee-friendly plant Thymus citriodorus on an urban extensive green roof. Acta Horticulturae 1298:575-580. https://doi.org/10.17660/ActaHortic.2020.1298.79 
Papafotiou M, Tassoula L, Mellos K (2018). Construction and maintenance factors affecting most the growth of shrubby Mediterranean native plants on urban extensive green roofs. Acta Horticulturae 1215:101-108. https://doi.org/10.17660/ActaHortic.2018.1215.18

Papafotiou M, Tassoula L, Liakopoulos G, Kargas G (2016). Effect of substrate type and irrigation frequency on growth of Mediterranean xerophytes on green roofs. Acta Horticulturae 1108:309-316. https://doi.org/10.17660/ActaHortic.2016.1108.41

Papafotiou M, Pergialioti N, Tassoula L, Massas I, Kargas G (2013). Growth of native aromatic xerophytes in an extensive Mediterranean green roof, as affected by substrate type and depth, and irrigation frequency. HortScience 48(10):1327-1333. https://doi.org/10.21273/HORTSCI.48.10.1327

Papafotiou M, Pergialioti N, Papanastassatos EA, Tassoula L, Massas I, Kargas G (2012). Effect of substrate type and depth and the irrigation frequency on growth of semi-woody Mediterranean species in green roofs. Acta Horticulturae 990:481-486. https://www.researchgate.net

Papafotiou M, Kargas G, Lytra I (2005). Olive-mill waste compost as a growth medium component for foliage potted plants. HortScience 40:1746-1750. http://hortsci.ashspublications.org

Perdetzoglou D, Kofinas C, Chinou I, Loukis A, Gally A (1996). A comparative study of eight taxa of Lomelosia RAF. (Dipsacaceae) from Greece, according to their fatty acid and sterol composition and antibacterial activity. Feddes Repertorium 107(1-2):37-42. https://onlinelibrary.wiley.com/doi/abs/10.1002/fedr.19961070107

Raven JA, Smith FA (1976). Nitrogen assimilation and transport in vascular land plants in relation intracellular $\mathrm{pH}$ regulation. New Phytologist 76:415-431. https://doi.org/10.1111/j.1469-8137.1976.tb01477.x

Reis M, Inácio H, Rosa A, Caccedilio J, Monteiro A (2001). Grape marc compost as an alternative growing media for greenhouse tomato. Acta Horticulturae 554:75-82. https://www.researchgate.net

Rowe B, Kolp MR, Greer S, Getter KL (2014). Comparison of irrigation efficiency and plant health of overhead, drip, and sub-irrigation for extensive green roofs. Ecological Engineering 64:306-313. https://doi.org/10.1016/j.ecoleng.2013.12.052

Said O, Fulder S, Khalil K, Azaizeh H, Kassis E, Said B (2008). Maintaining a physiological blood glucose level with 'Glucolevel', a combination of four anti- diabetes plants used in the traditional Arab herbal medicine. EvidenceBased Complementary and Alternative Medicine 5:421-428. http://dx.doi.org/10.1093/ecam/nem047

Said O, Khalil K, Fulder S, Azaizeh H (2002). Ethnopharmacological survey of medicinal herbs in Israel, the Golan Heights and the West Bank region. Journal of Ethnopharmacology 83:251-265. https://doi.org/10.1016/S0378-8741(02)00253-2

Sangüesa-Barreda G, Camarero JJ, Sánchez-Salguero R, Gutiérrez E, Linares JC, Génova M, ... López-Sáez JA (2019). Droughts and climate warming desynchronize Black pine growth across the Mediterranean Basin. Science of The Total Environment 697:133989. https://doi.org/10.1016/j.scitotenv.2019.133989

Santamouris M (2014). Cooling the cities - A review of reflective and green roof mitigation technologies to fight heat island and improve comfort in urban environments. Solar Energy 103:682-703. https://doi.org/10.1016/j.solener:2012.07.003

Santos M, Dianez F, Gonzalez del Valle M, Tello JC (2008). Grape marc compost: microbial studies and suppression of soil-born mycosis in vegetable seedlings. World Journal of Microbiology and Biotechnology 24(8):1493-1505. https://doi.org/10.1007/s11274-007-9631-0

Savi T, Andri S, Nardini A (2013). Impact of different green roof layering on plant water status and drought survival. Ecological Engineering 57:188-196. https://doi.org/10.1016/j.ecoleng.2013.04.048

Shackleton K, N Bell, H Smith L, Davies (2012). The role of shrubs and perennials in the capture and mitigation of particulate air pollution in London. Centre for Environmental Policy. Imperial College London. http://content.tfl.gov.uk/role-gi-pmpollution.pdf

Silva BR, Mantovani A, Mantuano DG, Rola SM, Barbosa MC (2018). Evaluating plant species suitability for a substratefree tropical green roof. Online Journal of Biological Sciences 18(4):401-423. https://doi.org/10.3844/ojbsci. 2018.401.423

Singh SP, Singh RP, Tiwari AK (2019). Evaluation of sugarcane varieties based on stomatal behavior under water stress condition. Sugar Technology 21:678. https://doi.org/10.1007/s12355-018-0680-5

Smaoui A, Barhoumi Z, Rabhi M, Abdelly C (2011). Localization of potential ion transport pathways in vesicular trichome cells of Atriplex halimus L. Protoplasma 248:363-372. https://doi.org/10.1007/s00709-010-0179-8 
Souza BD, Meiado MV, Rodrigues BM, Santos MG (2010). Water relations and chlorophyll fluorescence responses of two leguminous trees from the Caatinga to different watering regimes. Acta Physiologiae Plantarum 32:235244. https://doi.org/10.1007/s11738-009-0394-0

Stavrianakou S, Liakopoulos G, Miltiadou D, Markoglou AN, Ziogas BN, Karabourniotis G (2010). Antifungal and antibacterial capacity of extracted material from non-glandular and glandular leaf hairs applied at physiological concentrations. Plant Stress 4:25-30. https://www.academia.edu

Sun J, Gu J, Zeng J, Han S, Song A, Chen F ... Chen S (2013). Changes in leaf morphology, antioxidant activity and photosynthesis capacity in two different drought-tolerant cultivars of chrysanthemum during and after water stress. Scientia Horticulturae 161:249-258. https://doi.org/10.1016/j.scienta.2013.07.015

Tassoula L, Papafotiou M, Liakopoulos G, Kargas G (2015). Growth of native xerophytes Convolvulus cneorum L. on an extensive Mediterranean green roof under different substrate types and irrigation regimes. Hortscience 50(7):1118-1124. https://doi.org/10.21273/HORTSCI.50.7.1118

Tooulakou G, Giannopoulos A, Nikolopoulos D, Bresta P, Dotsika E, Orkoula MG ... Karabourniotis G (2016). Alarm photosynthesis: calcium oxalate crystals as an internal $\mathrm{CO}_{2}$ source in plants. Plant Physiology 171(4):2577-2585. https://doi.org/10.1104/pp.16.00111

Turner IM (1994). Sclerophylly: primarily protective? Functional Ecology 8:669-675.

Vardakas CI, Majchrzak D, Wagner KH, Elmadfa I, Kafatos A (2006). Lipid concentrations of wild edible greens in Crete. Food Chemistry 4:822-834. http://dx.doi.org/10.1016/j.foodchem.2005.08.058

Varone L, Ribas-Carbo M, Cardona C, Gallé A, Medrano H, Gratani L, Flexas J (2012). Stomatal and non-stomatal limitations to photosynthesis in seedlings and saplings of Mediterranean species pre-conditioned and aged in nurseries: Different response to water stress. Environmental and Experimental Botany 75:235-247. https://doi.org/10.1016/j.envexpbot.2011.07.007

Vijayaraghavan K (2016). Green roofs: A critical review on the role of components, benefits, limitations and trends. Renewable and Sustainable Energy Reviews 57:740-752. https://doi.org/10.1016/j.rser.2015.12.119

Walters SA, Stoelzle Midden K (2018). Sustainability of urban agriculture: vegetable production on green roofs. Agriculture 8(11):168. https://doi.org/10.3390/agriculture8110168

Ward D, Spiegel M, Saltz D (1997). Gazelle herbivory and interpopulation differences in calcium oxalate content of leaves of a desert lily. Journal of Chemical Ecology 23:333-346. https://link.springer.com/article/10.1023/B:JOEC.0000006363.34360.9d

Whittinghill LJ, Rowe DB, Andresen JA, Cregg BM (2015). Comparison of stormwater runoff from Sedum, native prairie, and vegetable producing green roofs. Urban Ecosystem 18:13-29. https://doi.org/10.1007/s11252-0140386-8

Whittinghill LJ, Rowe DB, Cregg BM (2013). Evaluation of vegetable production on extensive green roofs. Agroecology and Sustainable Food Systems 37(4):465-484. https://doi.org/10.1080/21683565.2012.756847

Whittinghill LJ, Rowe DB (2012). The role of green roof technology in urban agriculture. Renewable Agriculture and Food Systems 27(4):314-322. https://doi.org/10.1017/S174217051100038X

William R, Goodwell A, Richardson M, Le PVV, Kumar P, Stillwell AS (2016). An environmental cost-benefit analysis of alternative green roofing strategies. Ecological Engineering 95:1-9. https://doi.org/10.1016/j.ecoleng.2016.06.091

Xue M, Farrell C (2020). Use of organic wastes to create lightweight green roof substrates with increased plant-available water. Urban Forestry \& Urban Greening 48:126569. https://doi.org/10.1016/j.ufug.2019.126569

Yu L, Gao X, Zhao X (2020). Global synthesis of the impact of droughts on crops' water-use efficiency (WUE): Towards both high WUE and productivity. Agricultural Systems 177:102723. https://doi.org/10.1016/j.agsy.2019.102723

Zindler-Frank E (1991). Calcium oxalate crystal formation and growth in two legume species as altered by Strontium. Botanica Acta 104:229-232. https://doi.org/10.1111/j.1438-8677.1991.tb00222.x

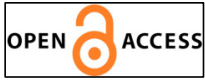

The journal offers free, immediate, and unrestricted access to peer-reviewed research and scholarly work. Users are allowed to read, download, copy, distribute, print, search, or link to the full texts of the articles, or use them for any other lawful purpose, without asking prior permission from the publisher or the author. 
Tassoula L et al. (2021). Not Bot Horti Agrobo 49(2):12283

(c) (P)

License - Articles published in Notulae Botanicae Horti Agrobotanici Cluj-Napoca are Open-Access, distributed under the terms and conditions of the Creative Commons Attribution (CC BY 4.0) License.

(c) Articles by the authors; UASVM, Cluj-Napoca, Romania. The journal allows the author(s) to hold the copyright/to retain publishing rights without restriction. 